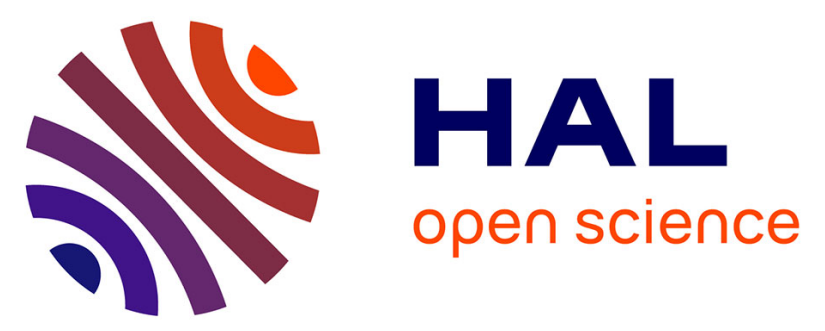

\title{
Numerical simulations of a fluidized granular flow entry into water: insights into modeling tsunami generation by pyroclastic density currents
}

Lily Battershill, Colin N. Whittaker, Emily M. Lane, Stéphane Popinet, James D. L. White, William L. Power, Paraskevi Nomikou

\section{To cite this version:}

Lily Battershill, Colin N. Whittaker, Emily M. Lane, Stéphane Popinet, James D. L. White, et al.. Numerical simulations of a fluidized granular flow entry into water: insights into modeling tsunami generation by pyroclastic density currents. Journal of Geophysical Research: Solid Earth, 2021, 10.1029/2021jb022855 . hal-03419617

\section{HAL Id: hal-03419617 \\ https://hal.science/hal-03419617}

Submitted on 8 Nov 2021

HAL is a multi-disciplinary open access archive for the deposit and dissemination of scientific research documents, whether they are published or not. The documents may come from teaching and research institutions in France or abroad, or from public or private research centers.
L'archive ouverte pluridisciplinaire HAL, est destinée au dépôt et à la diffusion de documents scientifiques de niveau recherche, publiés ou non, émanant des établissements d'enseignement et de recherche français ou étrangers, des laboratoires publics ou privés. 


\title{
Numerical simulations of a fluidized granular flow entry into water: insights into modeling tsunami generation by pyroclastic density currents
}

\author{
L. Battershill ${ }^{1,2}$, C. N. Whittaker ${ }^{1}$, E. M. Lane ${ }^{2}$, S. Popinet ${ }^{3}$, J. D. L. \\ White $^{4}$, W. L. Power ${ }^{5}$, P. Nomikou ${ }^{6}$ \\ ${ }^{1}$ Department of Civil and Environmental Engineering, University of Auckland, Auckland 1010, New \\ Zealand \\ ${ }^{2}$ National Institute of Water and Atmospheric Research, 10 Kyle Street, Christchurch, New Zealand, 8011 \\ ${ }^{3}$ Sorbonne Universit and CNRS, Institut Jean le Rond d'Alembert, UMR 7190, Paris, France \\ ${ }^{5}$ GNS Science, 1 Fairway Drive, Avalon, Lower Hutt 5011, New Zealand \\ ${ }^{4}$ University of Otago, 362 Leith Street, North Dunedin, Dunedin 9016, New Zealand \\ ${ }^{6}$ University of Athens, Liviis 19, Zografou 157 71, Greece
}

\section{Key Points:}

- A Newtonian fluid model is able to reproduce the wave generation mechanism at the interaction between a fluidized granular flow and water.

- The basal shear stress has a first-order control on the interaction dynamics and flow/wave energetics.

Corresponding author: Lily Battershill, 1bat537@aucklanduni.ac.nz

This article has been accepted for publication and ${ }^{-1}$ undergone full peer review but has not been through the copyediting, typesetting, pagination and proofreading process, which may lead to differences between this version and the Version of Record. Please cite this article as doi: 10.1029/2021JB022855.

This article is protected by copyright. All rights reserved. 


\begin{abstract}
The tsunami generation potential of pyroclastic density currents (PDCs) entering the sea is poorly understood, due to limited data and observations. Thus far, tsunami generation by PDCs has been modeled in a similar manner to tsunami generation associated with landslides or debris flows, using two-layer depth-averaged approaches. Using the adaptive partial differential equation solver Basilisk and benchmarking with published laboratory experiments, this work explores some of the important parameters not yet accounted for in numerical models of PDC-generated tsunamis. We use assumptions derived from experimental literature to approximate the granular, basal flow component of a PDC as a dense Newtonian fluid flowing down an inclined plane. This modeling provides insight into how the boundary condition of the slope and the viscosity of the dense granular-fluid influence the characteristics of the waves generated. It is shown that the boundary condition of the slope has a first-order impact on the interaction dynamics between the fluidized granular flow and water, as well as the energy transfer from the flow to the generated wave. The experimental physics is captured well in the numerical model, which confirms the underlying assumption of Newtonian fluid-like behaviour in the context of wave generation. The results from this study suggest the importance of considering vertical density and velocity stratification in wave generation models. Furthermore, we demonstrate that granular-fluids more dense than water are capable of shearing the water surface and generating significant amplitude waves, despite vigorous overturning.
\end{abstract}

\title{
Plain Language Summary
}

When a volcano erupts, it ejects large quantities of volcanic rock, gas, ash and debris. These ejected materials can flow very rapidly down the side slopes of the volcanothese flows are called pyroclastic density currents (PDCs). When PDCs enter the sea, they displace water and can generate tsunami waves with enormous destructive potential. One method of understanding this potential is by mathematically modelling the flow and its interactions with water, and confirming these model results against laboratory data. The present study compares numerical model results with published laboratory experiments of PDC generated tsunamis, to understand how our assumptions about the flow and its motion along the boundary can affect the amount of energy transferred to the generated waves. We approximate a PDC generated tsunami as a dense, viscous fluid moving down a slope into water. The amount of friction on the slope and the properties of the dense fluid lead to different interaction dynamics between the PDC and the water. The interaction dynamics lead to a wide range of wave breaking behaviours. Our results show the importance of the boundary conditions and fluid properties in correctly capturing experimental observations and in predicting how PDCs generate tsunamis.

\section{Introduction}

\subsection{Volcanic tsunamis}

Around $80 \%$ of tsunamis are triggered by underwater earthquakes which cause a sudden and rapid displacement of the water surface. Due to the wavelengths associated with the large horizontal scale of the fault rupture (tens to hundreds of $\mathrm{kms}$ ), this displacement results in long period waves capable of propagating across ocean basins (Center, 2006). Tsunamis can also be generated through sub-aerial and submarine landslides, meteorite impacts and volcanic eruptions. Volcanic eruptions themselves can generate waves through a number of mechanisms, including volcano-tectonic earthquakes, slope instabilities, pyroclastic density currents (PDCs), underwater explosions, shock waves and caldera collapse (Paris, 2015). There have been a number of geologically recent examples of such events. In 1996, the subaquatic explosive eruption near the northern shore of Karymskoye Lake in Kamchatka, Russia, generated multiple tsunamis (Belousov \& Belousova, 2000). Locally to the source $(r<1.3 \mathrm{~km})$, wave heights reached up to $30 \mathrm{~m}$ but were 
rapidly attenuated, leading to average runup heights of $2-3 \mathrm{~m}$ at locations $3 \mathrm{~km}$ from the source. Tsunamis generated by PDCs entering the sea were observed during the Montserrat 1997 and 2003 eruptions, with maximum run-up heights of $4 \mathrm{~m}$ in Montserrat (Narcisse et al., 2004), as well as the Rabaul 1994 eruption, where run-up heights reached $8 \mathrm{~m}$ in Rabaul Bay (Nishimura et al., 2000). The eruption of Krakatau volcano in 1883 triggered a tsunami that generated localized runup as high as $45 \mathrm{~m}$ and killed 36,000 people, understood to be as a result of voluminous PDCs entering the sea (Carey et al., 1996; Egorov, 2007; Maeno \& Imamura, 2011).

Globally, around $20 \%$ of deaths associated with erupting volcanoes are a result of tsunamis generated directly by the eruption (Center, 2006). Despite the fact that over half of these deaths are thought to be a result of pyroclastic density currents (PDCs) entering the sea, the tsunami generation potential of PDCs is still poorly understood. Not only are there limited observations, but experimental as well as theoretical studies are rare, due to the complexities involved in the modeling and observations of such phenomena (Paris, 2015).

Both the potential impact and the probability of occurrence of these mechanisms are often not included in tsunami hazard assessments, which are most often primarily focused on earthquake generated tsunamis. Coastal communities living close to active volcanoes may be unprepared for the possibility of tsunamis generated by volcanic eruptions (Paris, 2015). In 2002 a tsunami generated from lava and flank sliding at Stromboli volcano produced up to $10 \mathrm{~m}$ local runup and damage to buildings, but tourist facilities were seasonally unoccupied (e.g. Bonaccorso et al., 2003; Fornaciai et al., 2019). In July and August 2019, a sequence of pyroclastic density currents at Stromboli entered the sea, generating wave heights of up to $1 \mathrm{~m}$ near the entrance point (Giordano \& De Astis, 2021; Giudicepietro et al., 2020). Stromboli is only one of several coastal volcanoes that threaten the southern Tyrrhenian Sea. Another recent volcanic event which affected coastal communities was the December 2018 flank collapse of Anak Kraktau, Indonesia, which generated tsunami waves and killed over 400 people.

\subsection{Pyroclastic density currents}

PDCs are density currents made up of volcanic gas and particles. They are capable of transporting micrometer size ash particles to clasts larger than $1 \mathrm{~m}$ and can vary in temperatures from a few tens of ${ }^{\circ} \mathrm{C}$ up to $800^{\circ} \mathrm{C}$ (Sulpizio et al., 2014). Currents of interest in this study are ground-hugging, move at speeds of up to $100 \mathrm{~m} / \mathrm{s}$ down-slope away from their source (Legros \& Druitt, 2000; Freundt, 2003) and generally exhibit runout lengths of $10^{1} \mathrm{~km}$ or less. PDCs are among the most hazardous volcanic phenomena on Earth (Dufek, 2016; Lube et al., 2020). They form when hot mixtures of fragmented volcanic ash, rock and gas remain or become negatively buoyant with respect to the surrounding air, forming a particle-driven gravity current. PDCs originate by collapse of eruption columns (e.g. Sparks et al., 1978), by breakup and collapse of effusing domes above volcanic slopes (e.g. Ui et al., 1999), from inclined or laterally directed eruptive jets (e.g. Belousov et al., 2007) or from sustained pyroclastic fountaining (e.g. Báez et al., 2020). How PDCs form affects concentration, rheology and steadiness. For the present analysis of PDC-generated tsunamis, we focus on the currents typically generated on cone volcanoes, which commonly flow downslope and cross the shoreline (Edmonds \& Herd, 2005; Mattioli et al., 2007). PDCs are often subdivided according to their flow density (particle concentration). Lithofacies of PDC deposits reflect which type of flow emplaced them, and record transformations from one flow type to another (Fisher, 1979; Dufek et al., 2015; Lube et al., 2020):

- Dilute PDCs (pyroclastic surges), in which most of the mass and momentum are transported by a dilute, fully turbulent current, whose interaction with the substrate feeds a relatively thin bedload in which particles are transported by traction (e.g. Andrews \& Manga, 2012; Dellino et al., 2019; Fauria et al., 2016) 


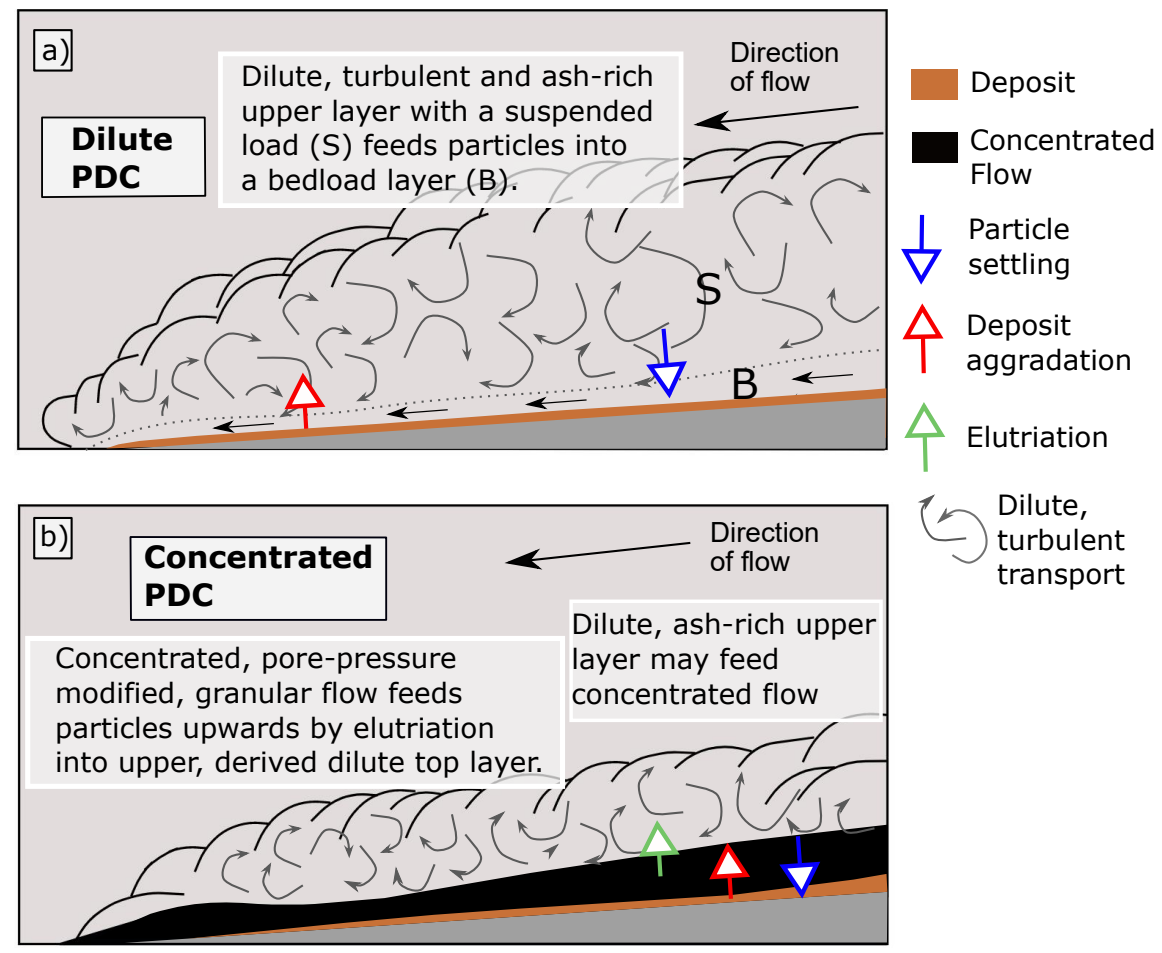

Figure 1. Diagram depicting the two key end-members of PDC: (a) a dilute PDC and (b) a concentrated PDC. The present study focuses on the concentrated end-member and ignores any momentum contribution from the dilute component.

- Concentrated PDCs (pyroclastic flows), comprising a gas-pore-pressure-modified granular flow (Wilson, 1980) overlain by a dilute cloud.

Figure 1 illustrates these two end members. Currents vary in velocity and temperature, and there are transitional regimes between these two end members, as well as downflow transformations from one to another. The boundary between dilute-upper and concentratedlower parts of a PDC may be diffuse, or may have a relatively sharp interface, i.e. a steep density gradient (Branney \& Kokelaar, 2005).

There are no direct observations of PDC interiors from the field, due to the hostile nature of the currents, the unpredictability of eruptive events and the dynamics of the events themselves (Baxter et al., 2005; Cas \& Wright, 1991; Legros \& Druitt, 2000). Experimental study of PDCs using particles and gas encompasses laboratory-scale studies of concentrated granular currents (Roche et al., 2002; Delannay et al., 2017; G. Smith et al., 2020) and dilute ones (Andrews \& Manga, 2012; Andrews, 2019), large-scale studies of hopper-fed confined currents (Lube et al., 2015, 2019, 2020) and of unconfined currents generated from experimental eruptions (Dellino et al., 2007, 2020). Of particular interest to our study, Freundt (2003) experimentally produce small, hot, pyroclastic flows that enter and interact with water.

The high mobility and near-frictionless behavior of PDCs observed both in the field and experimentally is an important characteristic of these currents, but understanding the source of this phenomenology is an ongoing area of research. While Lube et al. (2019) demonstrate the presence of an air-lubrication layer generated by an internal shear mechanism, other studies show that differential motion between particles and gas in the impact zone of a collapsing pyroclastic fountain can increase the pore fluid pressure within 
the PDC (Wilson, 1980; Roche et al., 2010; Valentine, 2020; Fries et al., 2021), which is then advected downstream by the flow, thus lowering the basal friction. Roche et al. (2008) show experimentally that pore-fluid pressure leads to Newtonian fluid-like behavior of fluidized granular flows. They conclude that efficient gas-particle interactions in dense, ash-rich pyroclastic flows may promote a water-like behavior during most of their propagation. The presence of pore-fluid pressure and the low-apparent basal friction coefficient of these currents are important considerations in the present study, when approximating the concentrated, basal component of a PDC.

The following section focuses on the ability of PDCs to generate tsunamis and outlines previous work on the subject including field studies and theoretical modeling, as well as numerical and experimental works.

\subsection{Pyroclastic density current generated tsunamis: current understand- ing and previous works}

Stratigraphic reconstruction, mapping of PDC deposits and observations of past events all suggest that in the past these currents have initiated tsunamis (e.g. Maeno \& Imamura, 2011; Nishimura et al., 2000; Nomikou et al., 2016; Sulpizio et al., 2014; Waythomas \& Watts, 2003). Geological investigation of sub-aqueous PDC deposits has concluded that when PDCs enter water they are generally disrupted explosively and/or ingest water and transform into water-supported mass-flows (e.g. Cas \& Wright, 1991; Jutzeler et al., 2017; Carey et al., 1996).

Theoretical studies also assume that PDCs are capable of passing into, over or under the water. Watts (2003) argues theoretically that the most energetic and coherent water waves are produced by the impact of the dense, basal, granular flow component of the PDC with water, assuming that the violent dynamics of the splash zone or vertical ejection of debris at interaction have negligible effects on wave generation. Other phenomena such as steam explosions, flow pressure, shear, and pressure impulse were considered, but the authors conclude that these mechanisms would generate smaller waves. Previous numerical works (e.g. Maeno \& Imamura, 2011; Nomikou et al., 2016), including the present study, accept this hypothesis and only consider the dense, basal component.

Laboratory experiments allow physical processes to be investigated in a controlled and (relatively) repeatable environment. This is particularly useful in the case of PDC modeling, where access to field data is limited. Furthermore, key parameters for numerical modeling must first be obtained from laboratory experiments.

Earlier experimental studies of tsunami generation by granular flows focus on initial parameters such as geometry and mass of an analog landslide (e.g. Fritz et al., 2003; Heller, 2009; Mohammed \& Fritz, 2012). The work of Fritz et al. (2003) explores landslide generated impulse waves and the associated generation of hydrodynamic impact craters. It identifies three different regimes associated with the interaction zone and shows that the amount (and rate) of water displacement is governed by the slide Froude number prior to impact (see Equation 2), the relative slide volume and the relative slide thickness (both with respect to the water depth). In the separated slide regime identified, a hydrodynamic impact crater forms, which is either outwards or backwards collapsing in nature.

Freundt (2003) addresses the interaction of a PDC with water, but primarily focuses on thermodynamic behaviour in the flow-water interaction zone. A series of experiments was conducted, where granular flows of heated ignimbrite ash $\left(20-400^{\circ} \mathrm{C}\right)$ and of bulk density near that of water, ran down a smooth chute and enter a water-filled tank at an angle of $26^{\circ}$. For lower temperatures, the majority of material penetrated the surface and mixed with water, creating a forward-directed ash fountain, a turbulent mixing zone and a water-supported mass flow. As the temperature was increased, most of the flow was redirected across the surface of the water, mixed with the water and generated steam explosions. No water-supported mass flow was generated in this latter case, 
but waves were generated as a result of steam explosions. Although waves were recorded during these experiments, their characteristics were not explored in detail.

More recent experiments on tsunami generation have pioneered research in the impact of cool, fluidized granular flows (representing the dense basal component of a PDC) into water and their effect on wave generation (Bougouin et al., 2020). Fluidized, micrometer $(\mathrm{d}=65 \pm 10 \mu \mathrm{m})$ spherical glass beads are released from a lock and propagate down a ramp (with a continuous supply of air flow to maintain pore fluid pressure), before interacting with water. This fluidization is used to replicate the high mobility/low friction behavior and the interstitial gas pore pressure of dense PDCs observed experimentally and in the field (e.g. Wilson, 1980; Roche et al., 2010; Lube et al., 2019; Valentine, 2020; Fries et al., 2021), while also helping to overcome scaling issues (Rowley et al., 2014; G. M. Smith et al., 2018). Notable features of the mixing zone include the generation of a vertical granular jet, a leading wave and a turbulent mixing zone, similar to that observed by Freundt (2003). The vertical granular jet redirects a small amount of material across the surface of the water, while the remaining flow forms a gravity current on the slope underwater. Spilling behaviour in the breaking wave is also observed. The equivalent experiments were conducted using dense salt water flows and yielded similar results to cases when fluidized grains were used. The Newtonian fluid-like behavior of fluidized granular flows proposed by Roche et al. (2008) is one possible explanation for this. Another reason for the similarity in results is the small particle size, which makes the granular flow nearly impermeable on impact.

According to previous works (e.g. Fritz et al., 2004; Heller, 2009; Zweifel et al., 2006), the wave amplitude associated with wave generation by granular-flows or rock-avalanches is primarily considered a function of a number of dimensionless parameters; the Froude number $F r$, the relative slide thickness $S$ and the relative slide mass $M$. The recent study of Bougouin et al. (2020) suggests a product impulse parameter $\zeta$ for waves generated by highly fluidized granular flows:

$$
\zeta=\left(\frac{u_{f}}{\sqrt{g H_{i}}}\right)\left(\frac{h_{f}}{H_{i}}\right)\left(\frac{\rho_{f}}{\rho_{0}}\right)\left(\frac{v}{H_{i}^{2}}\right) \sin \theta=F r S M \sin \theta
$$

with $u_{f}$ the front velocity at impact, $H_{i}$ the initial water depth, $h_{f}$ the height of the flow front $10 \mathrm{~cm}$ from the head at impact, $v$ the volume per width $v=\left(H_{0}-h_{r}\right) L_{i}$ (where $H_{0}$ is the initial column height, $h_{r}$ is the remaining granular flow in the reservoir and $L_{i}$ is the width of the reservoir), $\rho_{f}$ the effective density of the grains and $\rho_{0}$ the water density. The dimensionless amplitude scales as $A / H_{i}=f(\zeta)$, where $A$ is the wave amplitude in this setup $2.4 \mathrm{~m}$ from the shoreline. Bougouin et al. (2020) demonstrate how data for fluidized granular flows and dense salt water flows collapse onto the same curve, whereas dry granular flows show a notably lower amplitude for the same value of $\zeta$.

Bougouin et al. (2020) analyze features of the leading wave in the near-field region and it the primary conclusion is that in the case of fine-grained fluidized flows, the mass flux and volume of granular material are the primary parameters affecting the amplitude of the resulting wave. This is analogous to the findings from sub-aerial and submarine landslide literature, including Fritz et al. (2003) and the recent study by Robbe-Saule et al. (2020), which shows that the density has a second order effect on the wave amplitude.

Numerically modeling the interaction of a PDC with water and the resulting wave generation relies upon many simplifications. This includes approximating the density stratification and flow dynamics, as well as the sub-aqueous transport of the flow following its initial entry to the water. Previous numerical studies (e.g., Maeno \& Imamura, 2011; Nomikou et al., 2016) of PDC generated tsunamis assume the dilute component of a PDC to be negligible in terms of its effect on wave generation and focus on the dense, basal layer. Generally, these studies use depth-averaged approaches when considering both the $\mathrm{PDC}$ and the water (where vertical velocity stratification is ignored). Three dimensional numerical simulation can be used to capture the more complex physical processes occurring, but has been avoided in simulations of tsunami generation by PDC, primarily 
for computational efficiency when considering large scales. Capturing these physical processes is, however, a desirable next step towards improving our understanding of this phenomenon and improving the capabilities of present hazard assessment models.

In the context of volcanic landslide-generated tsunamis, the numerical benchmark study carried out by Esposti Ongaro et al. (2021) on the 2002 eruption of Stromboli, shows that the resulting waveform is highly sensitive to the source description. Furthermore, it shows that the use of non-hydrostatic models, coupled with a multilayer approach (where vertical velocity stratification is considered), allow for the most accurate hazard estimation. This highlights the importance of exploring similar levels of complexity in the context of PDC-generated tsunamis.

\subsection{Context of present study}

Modeling and predicting the behaviour of granular flows remains a challenging goal, since granular flows are characterized by a large diversity of behaviours depending on their environment and conditions (MiDi, 2004; Delannay et al., 2017). Creating a generic continuum granular rheology is still very much an active area of research, challenges including the identification of a relevant variable to describe the transition from arrest to flow and the understanding of non-local effects. A PDC adds further complexity, with basal friction effects and transient pore pressure complicating the modeling further (Breard et al., 2020). Lube et al. (2020) also note that the vertical velocity profile remains somewhat parabolic as well as transient. Furthermore, the velocity at the slope boundary is not necessarily zero and there is a broad range of velocity configurations within these currents.

The present study numerically models the interaction of a laboratory-scale dense PDC with water in a flume (the experiments of Bougouin et al. (2020)) and the associated waves generated using a two-dimensional numerical model, in order to investigate the potential of our model to capture some of the more complex physical processes occurring. This enables us to determine some of the key parameters involved in capturing the important physics. The definition of a boundary condition for the slope, in particular, is non-trivial. Our numerical study replicates the laboratory experiments of Bougouin et al. (2020), comparing with their experimental results and confirming that the adopted model is adequate to simulate laboratory experiments. Bougouin et al. (2020) propose that the granular flow can be approximated as a dense, single-phase Newtonian fluid from a wave generation perspective, which is a useful assumption to make numerically when simplifying the granular continuum rheology. Our numerical model is a useful means of testing this assumption. The modeling is achieved by numerically solving the NavierStokes equations on an adaptive grid, using the Basilisk flow solver (Popinet \& collaborators, 2013-2020). The granular-fluid is modelled as a Newtonian fluid, denser than water.

The numerical simulation outputs show a strong agreement with the experimental results. Figure 2 shows a direct comparison for different times, for two initial column heights and resulting granular-fluid Froude numbers. The Froude number for the granularfluid is defined as:

$$
F r=\frac{u_{f}}{\sqrt{g H_{i}}}
$$

where $u_{f}$ is the depth-averaged $u_{x}$ velocity over the height of the granular-fluid front at the moment of impact (or in the case of the laboratory experiments, the calculated front velocity), $H_{i}$ is the initial water depth and $g$ is the gravitational acceleration. In the numerical snapshots, we present two-dimensional vertical slices at the scale of the laboratory domain. The red represents the granular-fluid, the yellow the water and the blue the air. The granular-fluid is miscible in the water, but a sharp Volume of Fluid interface separates the granular-fluid and water from the air (see Section 2 for more details). Features of interaction including the generation of a granular jet, a plunging breaker and the retardation of the granular-fluid upon interaction with water are all captured in the numerical model. A characterization of interaction dynamics is discussed in Section 3.2 
$\mathrm{b} / \mathrm{H}_{0} \approx 0.11, \mathrm{Fr} \approx 1.8$
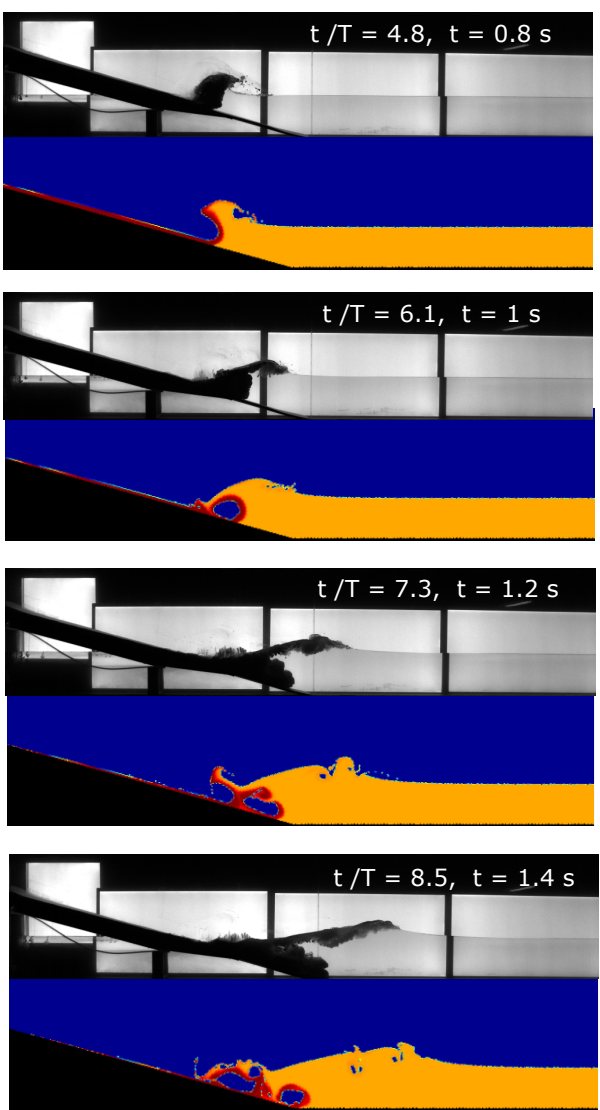

$\mathrm{b} / \mathrm{H}_{0} \approx 0.11, \mathrm{Fr} \approx 2.2$
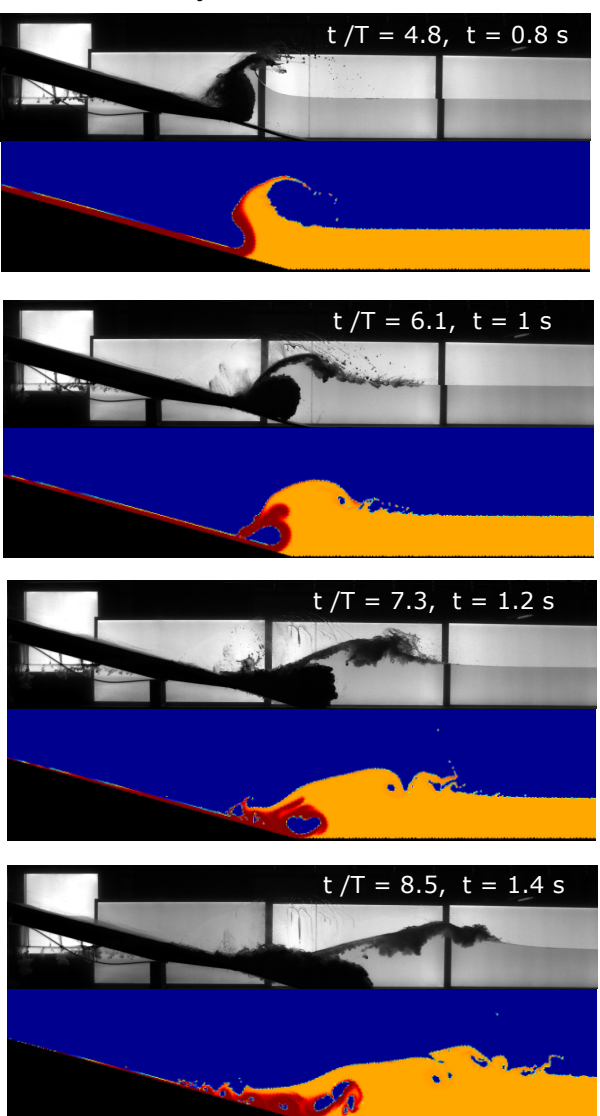

Figure 2. A comparison between numerical and experimental results (Bougouin et al., 2020), at four different times $t / T$ (where $\left.T=H_{i} / \sqrt{(} g H_{i}\right)$. The initial column heights in the experiments are $22.5 \mathrm{~cm}$ and $42.5 \mathrm{~cm}$ in the left and right columns, respectively. The numerical heights are initialized at $3 \mathrm{~cm}$ lower, to account for the residual grains left in the reservoir. The resulting Froude numbers are 1.8 and 2.2 in the left and right columns, respectively. More details on the setup information and outputs are discussed in methodology, Section 2.

This article is protected by copyright. All rights reserved. 
and a detailed discussion of the experimental/numerical comparison is presented in Section 3.3. The strength of agreement between the numerical results and the experiments presented is remarkable, but this is highly sensitive to the boundary condition. Hence, the present study also investigates the effect of variability in the boundary condition of the slope (i.e. the boundary friction) on the vertical (perpendicular to the slope) $x$ velocity profile of the granular-fluid, $u_{x}$, the wave generation process and the resulting farfield wave characteristics. A range of slope boundary conditions is explored and a detailed characterization of the associated granular-fluid/water interaction dynamics is presented. Furthermore, we investigate how different boundary conditions and associated interaction dynamics show different efficiencies of energy transfer from the granular-fluid to the water and the far-field wave. We first outline the methodology used, followed by an extensive discussion and presentation of our results in the following section.

\section{Methodology}

The following sections outline our numerical methodology. Section 2.1 gives the assumptions made and the governing equations solved, Section 2.2 provides details of the Basilisk flow solver and the numerical setup and Section 2.3 discusses the outputs analyzed.

\subsection{Assumptions made and governing equations solved}

We assume the fluidized grains from the experiments of Bougouin et al. (2020) to behave as a continuum. This takes the form of a dense, viscous and incompressible Newtonian fluid. The dense fluid and the water are assumed to be miscible with one another, but immiscible with air: they are separated from the air by a sharp interface. Surface tension is assumed to have negligible effect on interaction dynamics and wave propagation, due to the contrast of scales. The limitations of these assumptions are discussed in the discussion of results.

These assumptions lead to the applicability of the variable-density, multi-phase, incompressible Navier-Stokes equations:

$$
\begin{gathered}
\partial_{t} \mathbf{u}+\nabla \cdot(\mathbf{u u})=\frac{1}{\rho}\left[-\nabla p+\nabla \cdot\left(\mu\left(\nabla \mathbf{u}+\nabla \mathbf{u}^{T}\right)\right)\right]+\mathbf{g} \\
\nabla \cdot \mathbf{u}=0 \\
\partial_{t} f+\mathbf{u} \cdot \nabla f=0 \\
\partial_{t} c+\mathbf{u} \cdot \nabla c=D \nabla^{2} c
\end{gathered}
$$

with $p, \mathbf{u}, \mu, \rho, D$ and $\mathbf{g}$ representing the pressure field, velocity field, dynamic viscosity, density, diffusion coefficient and acceleration due to gravity respectively. $f$ is the volume fraction tracer in our VoF approach that delineates between air $(f=0)$ and the variable density fluid $(f=1)$. The density of the water/granular-fluid mixture can vary continuously between $\rho_{\text {water }}$ (where $c=0$ ) and $\rho_{\text {grains }}$ (where $c=1$ ). The same applies to the viscosity. The overall density $\rho$ and the viscosity $\mu$ are therefore functions of $c$ and $f$, i.e. $\rho(f, c), \mu(f, c)$. The diffusion term in Equation 6 expresses the diffusion of the granular fluid in water. In practice we set the diffusion coefficient D to zero but use a standard, diffusive numerical scheme (in contrast with the non-diffusive, geometric VOF scheme used to approximate Equation 5). The effective diffusion of the granular fluid in water is thus controlled by the properties of the numerical scheme and the spatial resolution. The method described is an alternative to an immiscible three-phase approach, where three fluids are separated by an interface (e.g., Joubert et al., 2020). 


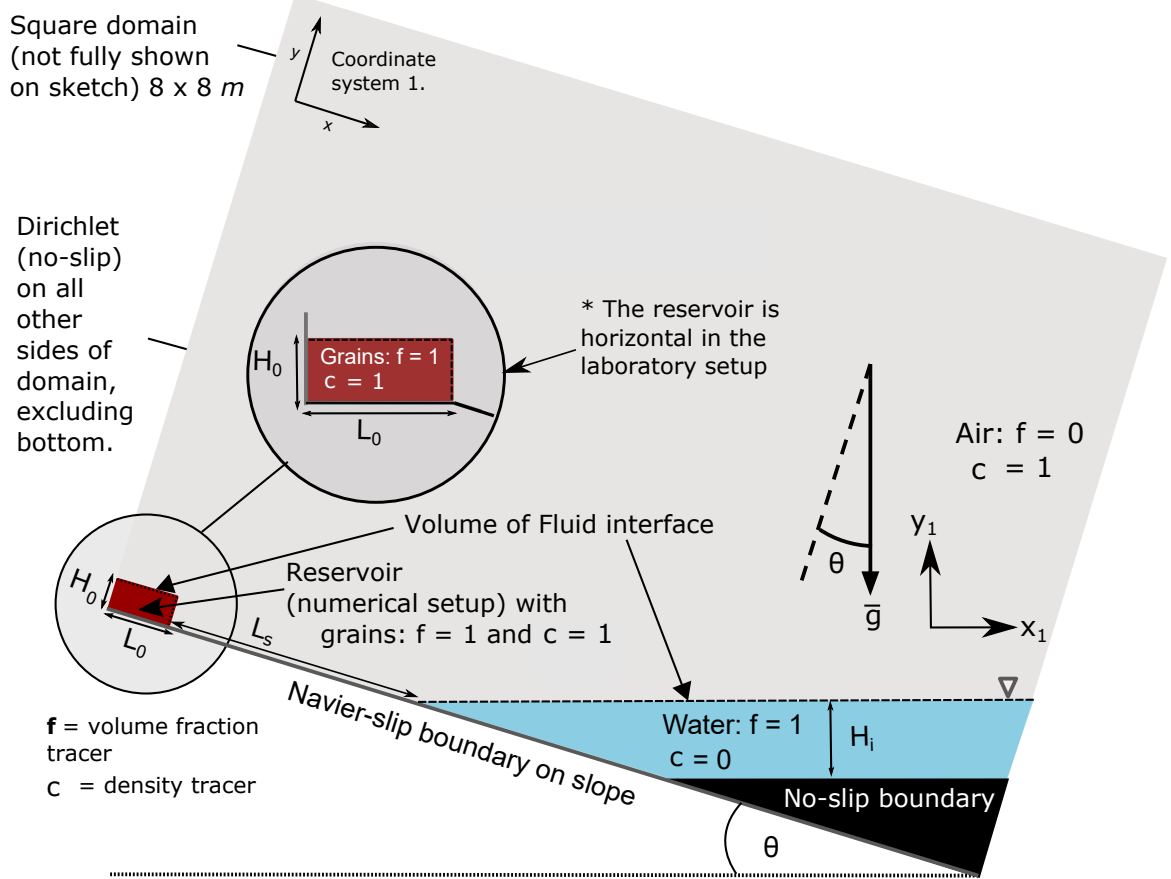

Figure 3. Setup of the initialized numerical domain, labelling the boundary implementations, tracer initialization and reference heights. The circular inset highlights the difference between the laboratory reservoir and the Basilisk initialization: the reservoir is horizontal in the laboratory setup, and tilted in the numerical setup. In our numerical simulations (and the physical experiments studied for comparison), $L_{0}=0.338 \mathrm{~m}, \theta=15^{\circ}, H_{i}=0.265 \mathrm{~m}$ and $L_{s}$ (length of exposed slope) $=1 \mathrm{~m}$.

\subsection{Numerical implementation}

Equations 3-6 are solved using the adaptive partial differential equation solver Basilisk (Popinet \& collaborators, 2013-2020), developed as the successor to Gerris by the same authors (Popinet, 2003, 2009, 2015). In Basilisk, an adaptive tree-grid structure is implemented which facilitates local refinement and coarsening, for computational efficiency. The Navier-Stokes solver has been successfully used in a number of two-phase problems to model splashing (Thoraval et al., 2012) and wave breaking in both two and three dimensions (Deike et al., 2015). A two-phase Volume of Fluid (VoF) approach is used to capture the interface between the air and the variable density fluid. Basilisk uses a conservative, non-diffusive, geometric VoF scheme (Scardovelli \& Zaleski, 1999). The consideration of a dense granular fluid (rather than a dilute phase) leads us to assume this single velocity model. The momentum equation is solved using the Bell Colella Glaz projection method (Bell et al., 1989), and we adapt the momentum-conserving scheme for $\mathrm{VoF}$ advection to account for variable density on the water/granular fluid side of the VoF interface.

In the present study we consider a two-dimensional vertical slice. This two dimensional approximation of a three dimensional process will lead to the generation of more coherent vortical structures, which has implications when considering wave breaking and overturning that must be considered. Since Basilisk works primarily with square or cubic domains, the length of the domain $L$ is set to be $8 \mathrm{~m}$ and the domain is rotated by angle $\theta=15^{\circ}$, to represent the slope (see Figure 3). This is in order to capture the dimensions of the experimental setup, see Bougouin et al. (2020) for details. This rotation 
of the domain leads to the definition of two coordinate systems; $x, y$ before rotation (where $x$ is in the downwards direction of the slope, with $y$ perpendicular) and $x_{1}, y_{1}$ after rotation. The bottom of the tank is implemented by masking the equivalent part of the numerical domain (i.e. setting the normal and tangential velocity components of each grid cell to zero).

A maximum grid resolution of $4096^{2}$ is used, leading to a minimum cell size of $L / 4096$ $\approx 1.4 \mathrm{~mm}$. For the initial column heights considered, this allows the vertical velocity profile of the granular-fluid and the interface boundary to be adequately resolved (see Figures S1 and S2 in supplementary material) .

In order to compare our results with the experiments of Bougouin et al. (2020), it is necessary to determine the parameters most representative of the experimental setup. The maximum density of the granular fluid is set to $1400 \mathrm{kgm}^{-3}$ and the density of the water is $997 \mathrm{kgm}^{-3}$. We have no information on the equivalent dynamic viscosity of the dense granular-fluid and the boundary condition on the slope in the experimental setup, since these conditions are non-trivial to define. Section 3 therefore presents an exploration of this parameter range (and the associated granular-fluid velocity profiles) in order to determine the most representative conditions and to explore how these parameters control granular-fluid/water interaction dynamics. We choose a range of viscosities which lead to similar impact velocities to those observed in the laboratory experiments. This comparison gives us a benchmark against which to check depth-averaged or multi-layer approaches (e.g., Audusse, 2005; Popinet, 2020).

We choose to explore a range of friction (boundary) conditions within our numerical model for the granular-fluid. We therefore use a Navier-slip boundary for the slope boundary condition, viz.,

$$
u_{t}+b \frac{\partial u_{t}}{\partial z}=0
$$

where $b$ represents the Navier-slip length of the granular-fluid. The choice of this Navierslip length allows us to vary this boundary condition between no-slip, partial-slip and free-slip. Varying the boundary condition between the free-slip and no-slip end members allows us to explore the full range of slip lengths to better constrain the model.

The Navier-slip boundary condition is set along the bottom $x$ boundary (i.e. the slope). For the implementation of the tank bottom, the velocity field is set to zero at all time-steps, leading to a no-slip (Dirichlet) boundary condition, as depicted by the shaded black area in Figure 3. This implementation is limited by the current capabilities of embedded boundaries in Basilisk, however the primary focus of our analysis is associated with initial wave generation and propagation before the current interacts with the bottom boundary. The vertical $u_{x}$ profile of the granular-fluid is dependent on the granularfluid viscosity and boundary condition. The boundary-layer thickness (denoted in Figure 4 by $\delta_{x}$ ) represents the distance normal to the wall to a point where the velocity of the granular-fluid has reached a certain percentage of the outer velocity $u_{\max }$, e.g. $99 \%$. (Schlichting \& Gersten, 2016). There is no unique boundarylayer thickness, since the effect of the viscosity in the boundary layer decreases asymptotically as we move outwards from the wall.

\subsection{Outputs}

Following Bougouin et al. (2020), we evaluate the front height $h_{f}$ and output the front velocity profile $u_{x, \text { front }}$ at $10 \mathrm{~cm}$ from the head of the granular-fluid at the time of impact (i.e. $10 \mathrm{~cm}$ from the slope-water intersection). The constant front velocity $u_{f}$ is defined in our numerical experiments as the depth-averaged velocity at this location. We also consider the energy of the system. We calculate the total energy:

$$
E=E_{k}+E_{g}
$$


as the sum of gravitational potential energy;

$$
E_{g}=\int \rho g y d x d y-E_{\text {rest }}
$$

and the kinetic energy:

$$
E_{k}=\frac{1}{2} \int \rho u^{2} d x d y
$$

for the granular-fluid, water and air. The components are calculated at each location using the respective volume fraction $f$ and granular-fluid tracer $g$ values. At initialization, the kinetic energy is 0 and the total energy of the domain is stored in the potential energy of the granular-fluid, i.e. $E_{\text {init }}=E_{g, \text { init }}$. The constant $E_{\text {rest }}$ is the minimal gravitational potential energy achievable by the system, i.e. the potential energy corresponding to a horizontal layer of granular fluid overlaid by a horizontal layer of water.

\section{Results}

\subsection{Vertical profiles of the horizontal velocity component of the granular- fluid, at impact}

Figure 4 shows the vertical profiles of the horizontal velocity component of the granularfluid at impact (the time-step at which the granular-fluid first interacts with the water) as we vary the boundary between the no-slip and free-slip end members. Velocity profiles are shown for two different values of dynamic viscosity: $\mu=0.01 \mathrm{~Pa} s$ and $\mu=0.1$ $P a s$, for initial column heights $H_{0}=18.5 \mathrm{~cm}$ and $H_{0}=39.5 \mathrm{~cm}$. The dimensionless slip length is defined as: $b / H_{0}$.

For a given dynamic viscosity $\mu$ and given column height $H_{0}$, the thickness of the boundary layer $\delta_{x}$ at impact remains approximately the same for all values of slip length $b$, whereas the depth-averaged velocity across the flow front $u_{f}$ is highly dependent on $b$. As the dynamic viscosity of the granular flow increases, the boundary layer thickness $\delta_{x}$ increases. As the initial column height $H_{0}$ increases, the flow front height $h_{f}$ at impact also increases, but only a small increase of $\delta_{x}$ is observed. For higher dynamic viscosities and lower initial column heights (i.e. $\mu=0.1 \mathrm{~Pa} \mathrm{~s}, H_{0}=18.5 \mathrm{~cm}$ ), we observe a well-resolved boundary layer, as shown in Figure 4. As dynamic viscosity is reduced (i.e. $\mu=0.01 P a s$ ), a higher resolution is required to resolve a similar number of grid cells over the boundary layer.

\subsection{Snapshots of interaction dynamics}

Longer slip lengths (higher basal slip velocities) exhibit notably different interaction dynamics between the granular-fluid and the water, compared with shorter slip lengths. This leads to different wave generation mechanisms associated with different slip lengths, as illustrated in Figure 5. The interaction dynamics depicted are present across the range of column heights and dynamic viscosities considered, however Figure 5 presents snapshots from the case where $\mu=0.1 P a s$ and $H_{0}=18.5 \mathrm{~cm}$.

The interaction dynamics are differentiated qualitatively by the amount of granularfluid directed across the water surface versus down-slope, and the resulting breaking wave characteristics. The breaking characteristics are described by the amount of overturning of the free surface.

At the no-slip end-member (where the basal slip velocity is equal to zero), the granularfluid is generally directed across the water surface at impact and the breaking wave most often exhibits violent overturning (Figure 5 subfigures a-c). No water surface closure behind the wave front is observed in this case and water rushes back towards the ramp under the influence of gravity. This type of collapse behind the leading wave is referred to 

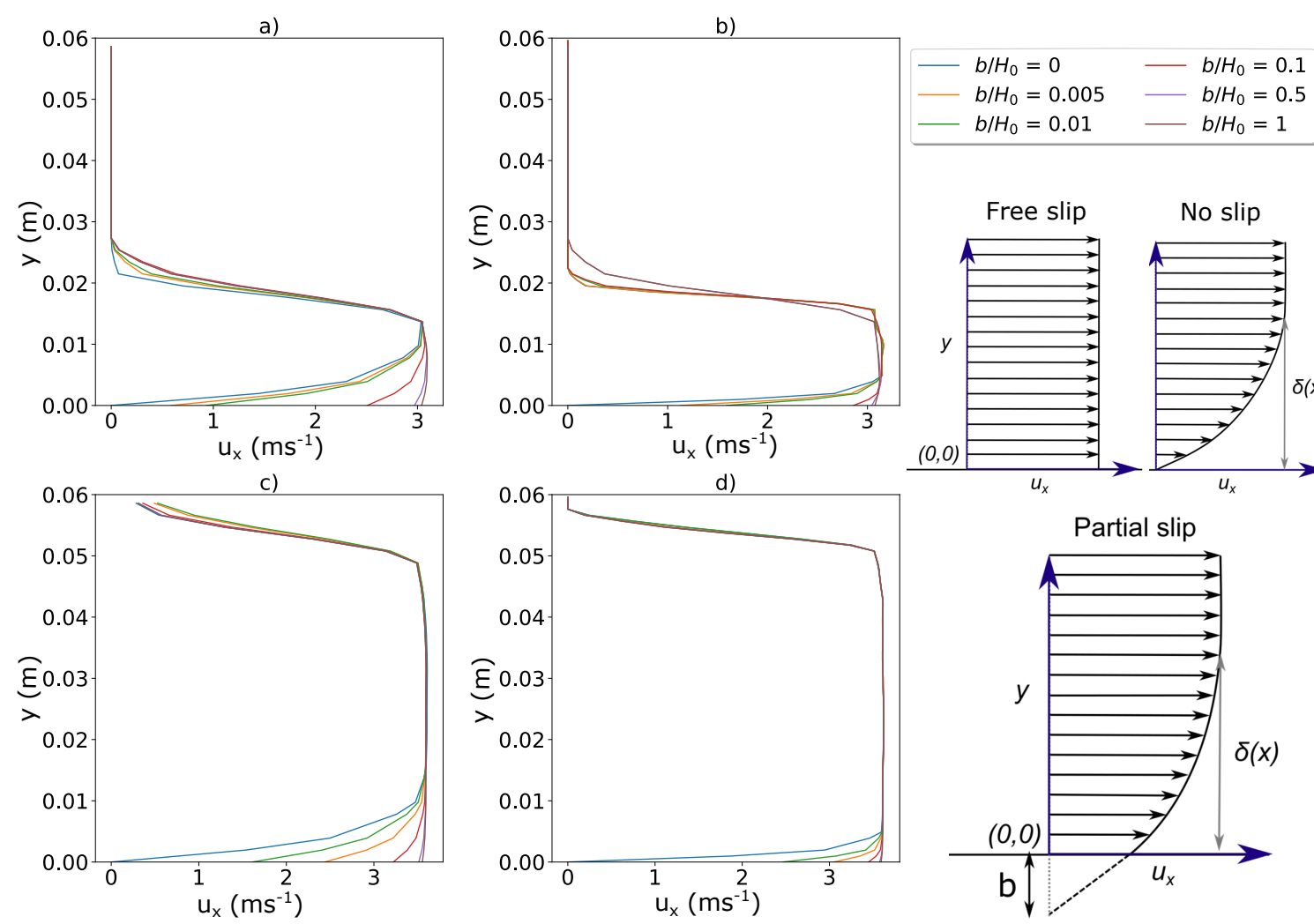

Figure 4. Velocity profiles of the granular-fluid (10 $\mathrm{cm}$ from the head) at the time of impact, for a range of dimensionless slip lengths $b / H_{0}$. a) $\left.H_{0}=18.5 \mathrm{~cm}, \mu=0.1 \mathrm{~Pa} \mathrm{~s}, \mathrm{~b}\right) H_{0}=18.5 \mathrm{~cm}$, $\mu=0.01 \mathrm{~Pa} \mathrm{~s}, \mathrm{c}) H_{i}=39.5 \mathrm{~cm}, \mu=0.1 \mathrm{~Pa} \mathrm{~s}$, a) $H_{0}=39.5 \mathrm{~cm}, \mu=0.01 \mathrm{~Pa} \mathrm{~s}$. Light blue inset shows how boundary conditions on the slope affect the boundary layer thickness $\delta_{x}$ at a time $t$. Graphical depiction of slip length $b . b=0$ for no-slip and $b=\infty$ for free-slip. 


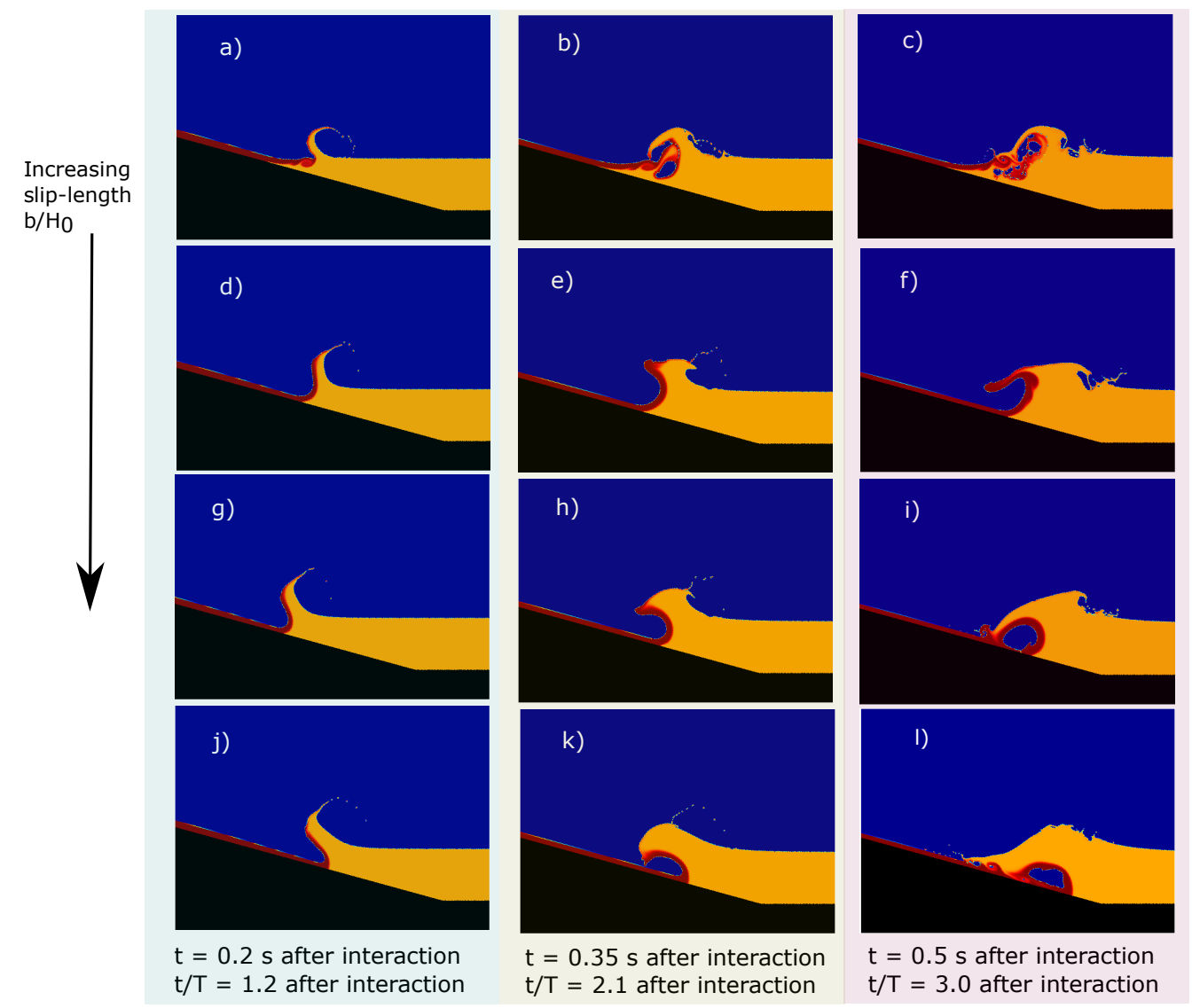

Figure 5. These snapshots are for the initial conditions $\mu=0.1 \mathrm{Pas}$ and $H_{0}=18.5 \mathrm{~cm}$. The $b / H_{0}$ values shown are specific to these initial conditions, leading to $\mathrm{Fr}=1.8$ (Equation 2 ). a) c) No-slip/low values of slip: the granular-fluid shears the water surface leading to violent overturning. d) - f) Low to medium values of slip: the granular-fluid partly shears the water surface, and partly propagates downslope, leading to a steep, plunging breaker. g) - i) Medium to high values of slip: most of the granular-fluid is directed downslope, with some shearing of the water surface. This leads to the generation of a plunging breaker. j) - 1) Free slip: granular-fluid fully propagates downslope, with little/no overturning in resulting wave. 
as an outwards collapsing impact crater, considering the terminology used in the landslide tsunami generation experiments of Fritz et al. (2003). This type of interaction does not generally support the immediate generation of a gravity current and most material therefore remains near the interaction zone. In some cases, however, at the no-slip endmember, the granular-fluid lifts fully off the bottom boundary and then re-attaches to the ramp. This reattachment reduces the amount of overturning observed in the breaking wave. Figure S3 in the supplementary material demonstrates an example when noslip leads to reattachment and the generation of a gently spilling wave (Figure S3 subfigures a-c).

For higher basal-slip velocities, more granular-fluid becomes directed down-slope rather than across the water surface. This leads to the generation of a plunging breaker (Figure 5 subfigures d-f). As the basal-slip velocity is increased further, the wave steepness and amount of overturning/breaking decreases. Under these conditions, a backwards collapsing impact crater is observed (Fritz et al., 2003). This type of impact crater is governed by a surface closure resulting in the inclusion of air in the form of a cavity.

At the free-slip end-member (where the basal slip velocity is equivalent to the free stream velocity), the granular-fluid appears to initially expel the water upwards, then punches through the water, also forming a backwards collapsing impact crater (Figure 5 subfigures g-i). A spilling breaker is generated from the initial uplift, showing no significant breaking overturning.

For each viscosity and column height, the change in interaction dynamics generally follows the same pattern as the dimensionless slip length $b / H_{0}$ is increased. Figure $\mathrm{S} 3$ in supplementary material shows the equivalent of Figure 5 , for $H_{0}=39.5 \mathrm{~cm} \mu=$ $0.01 \mathrm{~Pa} \mathrm{~s}$. The values of $b / H_{0}$ corresponding to the transition between different interaction dynamics vary depending on the Froude number of the granular-fluid (see Equation 2), but qualitatively similar dynamics are observed.

These results and observations imply that the boundary condition plays an important role in determining the interaction dynamics and the characteristics of the generated wave.

\subsection{Experimental comparison}

Figure 2 in Section 1.4 demonstrates excellent qualitative agreement between the observed behaviour in the numerical simulations and physical experiments. Sections 3.1 and 3.2 show how the boundary condition influences this agreement, demonstrating the sensitivity of the velocity profiles and granular-fluid/water interaction dynamics to the choice of boundary condition. In Section 3.3.1, we explore the qualitative comparisons in more detail and discuss the limitations of the numerical model in capturing the experimental physics. Section 3.3.2 expresses the numerical results in terms of the product impulse parameter (Equation 1), and shows the applicability of this parameter as a predictor of the dimensionless wave amplitude in our simulations. This also provides a quantitative measure of the agreement between the numerical results and laboratory measurements over a range of boundary conditions, viscosities and column heights.

\subsubsection{Granular-fluid/water interaction dynamics}

The experimental results cover a range of Froude numbers comparable to our numerical simulations, information related to the friction condition is limited. In the laboratory experiment snapshots (Bougouin et al., 2020), the details of the interaction behind the granular-fluid front (e.g. evidence of a hydrodynamic impact crater) cannot be observed due to vigorous mixing of the granular material. However, a number of key features associated with the wave generation, the granular-fluid separation and the propagation of the gravity current may still be identified. As the granular-fluid impacts water, some momentum is directed across the water surface, causing the generation of an outwards projecting granular jet. This behaviour is also reported in the cool volcanic ash 
experiments of Freundt (2003). The majority of the granular-fluid undergoes mixing after impact and forms a water-supported mass flow which travels down-slope, at a slower velocity to that of the leading wave. For experimental Froude numbers $>2.0$, the wave generated in the initial 0.4 seconds after interaction displays features of a steeply plunging breaker. As the Froude number decreases, the plunging breaker becomes increasingly gentle and for lower Froude numbers (e.g. Fr $\approx 1.6$ ), a spilling breaker is generated. The Froude number considered uses the granular-fluid front velocity at the moment of impact (i.e. prior to mixing and deceleration) with respect to the shallow water wave speed (see Equations 1 and 2).

Figure 5 shows that high values of partial-slip best reproduce the interaction dynamics observed in the laboratory experiments. Work on granular flows with pore fluid pressure and/or on inclines shows that there is significant slip on a smooth substrate (e.g. Roche et al., 2010; Brodu et al., 2015; Lube et al., 2019), so this inference is in line with these experimental observations.

Figure 2 shows directly how the numerical results (for a high value of partial slip, $\left.b / H_{0}=0.1\right)$ capture the generation of the granular jet, the plunging breaker behaviour with associated splashes and overturning, as well as the approximate shape and velocity of the gravity current in the first $1.2 \mathrm{~s}$.

While the overall comparison is favourable, there are some discrepancies. At the interaction zone, where the numerical results show the formation of an impact crater, the experimental snapshots show vigorous mixing. Furthermore, the numerical results show increased breaking and overturning of the breaking wave at later time-steps in comparison with the experimental snapshots. We attribute these discrepancies to the limitations surrounding the immiscibility of air and granular-fluid in our simulations, and the two-dimensional representation of an intrinsically three-dimensional process. Given that our model assumes the grains to behave as a continuum (in the form of a Newtonian fluid) confined to one side of the interface, we do not capture the dynamics of individual grains and we therefore do not capture the same level of mixing observed in the physical experiments. This means the numerical model is likely to overemphasize the formation of an air pocket/cavity. Furthermore, the two-dimensionality of this model leads to enhanced vortical structures, in both the interaction zone and the breaking wave.

In addition, after the gravity current reaches the base of the tank, some lift is observed from the boundary in our numerical simulations. This is likely a result of the bottom (horizontal) boundary implementation, which is limited to no-slip. As shown in the interaction of the granular-fluid with water for no-slip conditions, granular-fluid lift is often observed. These limitations do not impact our overall conclusions, however, and the numerical/experimental agreement is of good quality. The wave generation appears qualitatively similar and the propagation and shape of the leading wave are well captured.

In the high temperature experiments of Freundt (2003), all of the granular-fluid is redirected across the surface of the water, leading to violent overturning, similar to what is observed in Figure 5 a-c. Localized waves of smaller amplitude are observed, which are associated with steam explosions occurring near the surface of the water. Although temperature is not considered in our numerical simulations, we observe a similar interaction behaviour for the no-slip end member, whereby the granular-fluid is redirected across the surface of the water, leading to violent breaking behaviour. This granular-fluid redirection can be attributed to a number of potential factors, including changes in density or buoyancy, boundary behaviour and shear. The present study does not explore the effects of temperature, but this is an interesting area for future research.

\subsubsection{Product impulse parameter and dimensionless wave amplitude}

We compare our numerical results with the experiment results of Bougouin et al. (2020) using the product impulse parameter $\zeta$, which they showed to be a good predictor of the dimensionless wave amplitude (Figure 6). Our numerical data match the laboratory results well over a range of column heights, which provides confidence in the nu- 


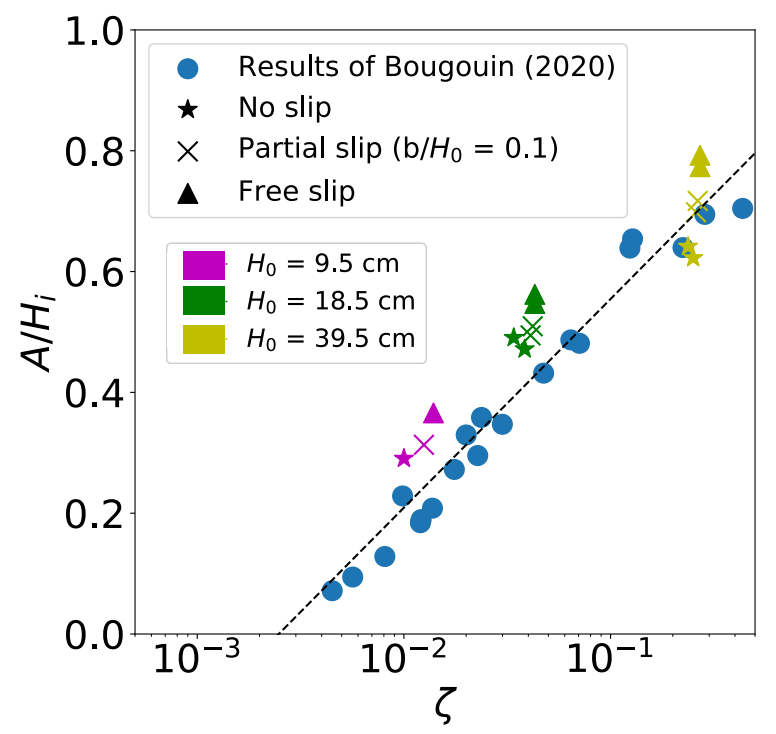

Figure 6. Normalized amplitude $A / H_{i}$ at $x_{1}=2.4 \mathrm{~m}$ from the shoreline as a function of the impulse parameter $\zeta$ (Equation 1), for the laboratory experiments (of fluidized glass beads) and the numerical experiments considered in the present study. Results from the numerical simulations are shown for multiple slip conditions and initial column heights (represented by the marker style and color, respectively). For $H_{0}=39.5 \mathrm{~cm}$ and $H_{0}=18.5 \mathrm{~cm}$, markers are shown for both $\mu=0.1 P a s$ and $\mu=0.01 P a s$. Dotted line (- $) A / H_{i}=0.15 \ln (\zeta)+0.88$.

merical model (and therefore the Newtonian-fluid approximation of the granular-fluid). The numerical wave amplitudes are slightly higher than the experimental results; this is likely due to a number of factors, including the two-dimensional approximation of threedimensional turbulence, the differences in flow rheology, the immiscibility between the granular-fluid and air, and the difference in reservoir geometry (see Figure 3). Furthermore, subtle differences between the numerical and laboratory methodologies for calculating $u_{f}$ (we consider depth-averaged velocity over the height of the flow $h_{f}$, where the laboratory experiments use the constant flow font velocity) may lead to small differences in $\zeta$. Finally, the calculation of wave amplitude in both experimental and numerical methodologies has a degree of uncertainty.

Across the range of initial conditions considered in our numerical results, a change in boundary condition leads to a considerable difference in the resulting wave amplitude. Partial-slip boundary conditions lead to wave amplitudes closer to the laboratory data points, compared with free-slip conditions. This agrees with our qualitative inferences, which show partial slip conditions to better capture the interaction dynamics observed in the laboratory snapshots. Free-slip conditions lead to little to no breaking (Figure 5 $\mathrm{a}-\mathrm{c})$, which is one reason for these larger amplitudes.

While the inferences drawn from this Figure are useful, further analysis on far-field $\left(x_{1}>5 m\right.$ from the shoreline) amplitudes and overall energetics is required to constrain further the effect of boundary condition (see Section 3.4).

\subsection{Energy transfer and wave characteristics}

\subsubsection{Energy evolution and transfer}

It has been demonstrated across multiple experimental and numerical studies (e.g., Deike et al., 2015) that wave breaking has a significant effect on energy dissipation and 
a)
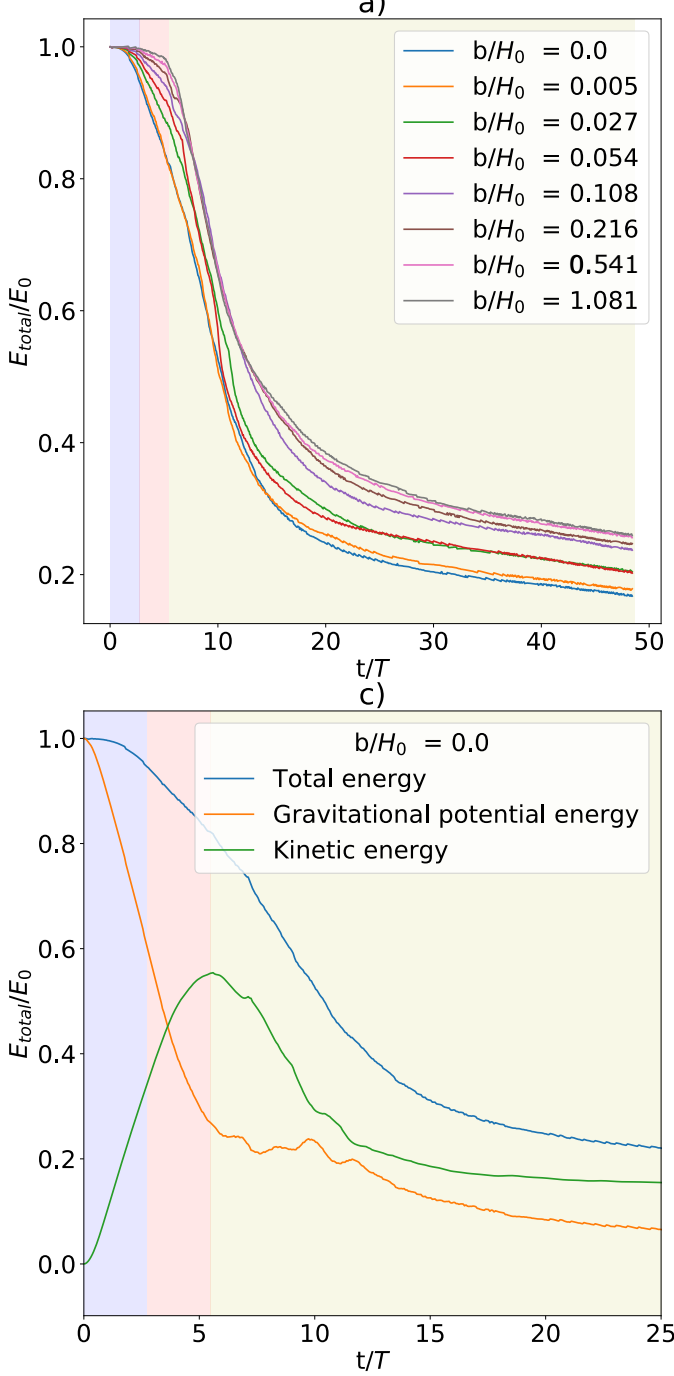

b)

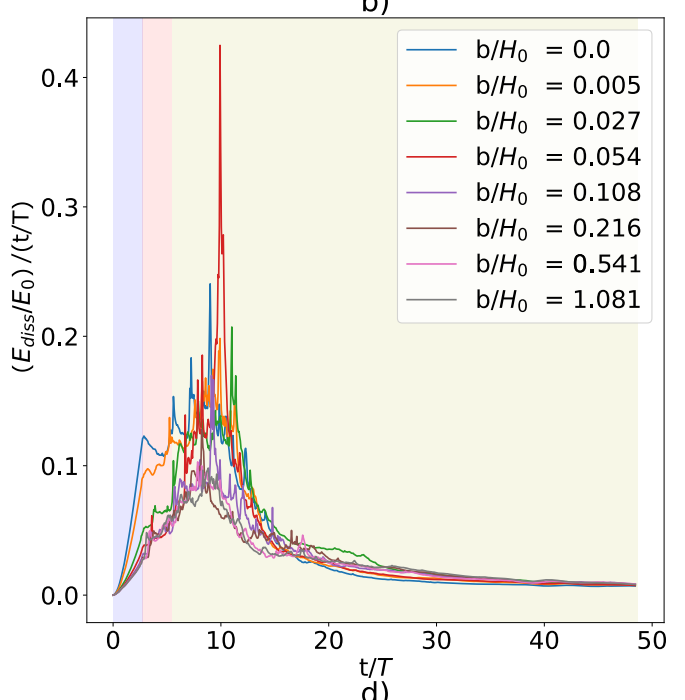

d)

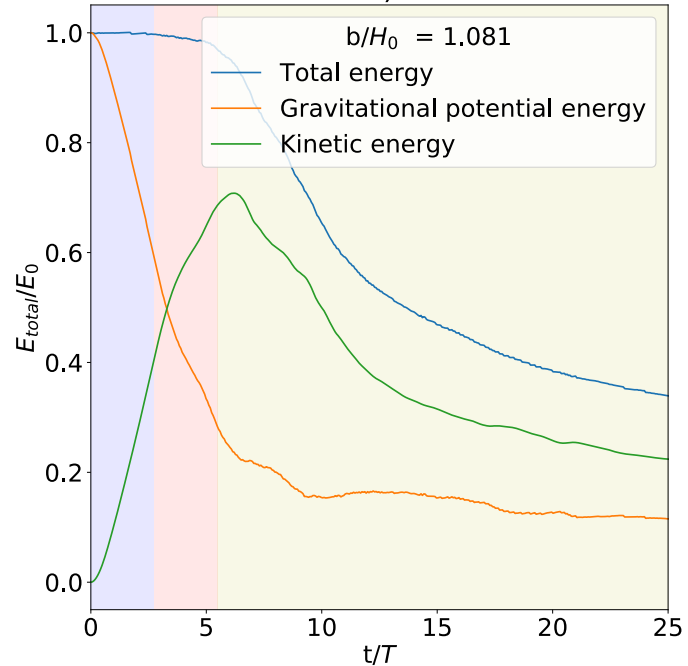

Figure 7. a) Normalized total energy of domain as a function of time $t / T$ (where $T=$ $H_{i} / \sqrt{ }\left(g H_{i}\right)$, for a range of slip lengths $b / H_{0}$. b) Energy dissipation rate (relative energy lost per $T$ ) of entire domain as a function of time $t / T$. c) and d) Normalized total energy of domain, including the normalized gravitational and kinetic components as a function of time $t / T$ for $b / H_{0}$ $=0.0$ and $b / H_{0}=1.081$. The background colors broadly represent the different stages of the simulation: purple $=$ granular-fluid propagation on slope prior to interaction, pink $=$ interaction and initial wave generation and yellow = wave breaking and impact crater collapse. 
momentum transfer. In our numerical simulations there are a number of significant dissipation processes occurring, including wave breaking, collapse of the hydrodynamic impact crater, and air entrainment at the mixing zone. In the case where a spilling wave is observed and less dissipation associated with wave breaking would be expected (for some of the high slip conditions, no breaking is observed), there may be increased dissipation elsewhere in the domain; i.e. in the impact crater collapse or the propagation of the gravity current. Similarly, in the case of violent overturning there is no significant impact crater collapse or propagation of a gravity current. Thus, beyond the initial granularfluid propagation and water impact, differentiating between energy dissipation mechanisms is non-trivial. Exploring the energy evolution of the domain (and its components) does, however, allow us to determine the amount and timing of energy dissipation associated with the different boundary conditions and the relative granular-fluid/water interaction dynamics.

Figure 7 a shows the evolution of the normalized total energy of the domain and Figure $7 \mathrm{~b}$ shows the dissipation rate for a range of slip lengths $b / H_{0}$, for the initial condition where $H_{0}=18.5 \mathrm{~cm}$ and $\mu=0.1 \mathrm{~Pa} \mathrm{~s}$. Figures $7 \mathrm{c}$ and $7 \mathrm{~d}$ present the evolution of the normalized total energy of the domain, along with the gravitational and potential components, for $b / H_{0}=0.0$ and $b / H_{0}=1.081$. Figure 8 presents the kinetic, potential and total energy evolutions for the granular-fluid and the water components, as well as their dissipation rates. Similar evolution patterns can be seen across a range of initial conditions; an example for $H_{0}=39.5 \mathrm{~cm}$ and $\mu=0.01 \mathrm{~Pa} s$ is shown in the supplementary material (Figure S4).

As the granular-fluid propagates down the slope, the total energy of the domain begins to decrease. This decrease is greater for high friction cases (i.e. smaller values of $\left.b / H_{0}\right)$ and is most clearly depicted in the total energy dissipation plot (Figure $7 \mathrm{~b}$ ), which shows the increased dissipation rate for high friction conditions during the initial propagation. Figures $8 \mathrm{~b}$ and $8 \mathrm{~d}$ show the transfer of potential energy to kinetic energy within the granular-fluid as it propagates down-slope, with Figure 8h showing that the dissipation occurring at this stage is driven by the granular-fluid.

As the granular-fluid impacts the water and the water surface is uplifted (as depicted in Figure $5, t / T=1.2$ after interaction), the total energy of the domain continues to decrease for all values of $b / H_{0}$. At the transition between uplift and the onset of wave breaking (and/or impact crater collapse), the kinetic energy of the domain and the kinetic energy of the granular-fluid reach a maximum for all values of $b / H_{0}$ and the rate of change in potential energy decreases, as the granular-fluid slows down within the collapsing region (i.e. Figure $5, t / T=2.1$ after interaction). When the granular-fluid shears the water surface, the potential energy of the granular-fluid remains higher than for other interaction styles. The maximum value of total kinetic energy observed is $\approx 20 \%$ higher for larger slip conditions (i.e. $b / H_{0}=1.081$ ), since in these cases less energy has been dissipated in the initial propagation, interaction and wave generation stages. Once wave breaking starts and/or the impact crater collapses, the granular-fluid is slowed and the kinetic energy of the granular-fluid decreases abruptly, which corresponds with a decrease of the total energy in the domain. However, during this stage, the total energy of the water and its components continue to increase. Generally, the dissipation rate at this stage increases as the amount of overturning increases in the wave breaking and entrainment of air at the shoreline during the impact crater collapse.

The total energy of the water reaches a maximum at $t / T \approx 10$ and is greatest for lower friction conditions (see Figure 8e). The time of maximum energy in the water corresponds to the time at which the dissipation rate of the granular-fluid begins to slow, and the potential energy of the granular-fluid flattens, suggesting that the grains stop imparting significant energy to the water at this stage. It can be observed that after $t / T$ $\approx 20$, the energy dissipation rates for all slip conditions begin to tend towards a steady rate, as breaking ceases and the granular-fluid has undergone significant mixing.

For later times (i.e. $t / T>20$ ), the total energy of the water tends towards a more constant value. When considering tsunami generation potential, this observation sug- 
a)

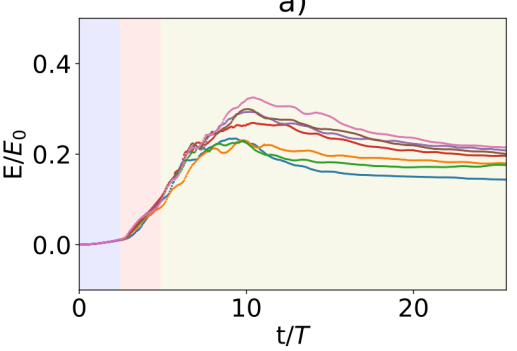

c)

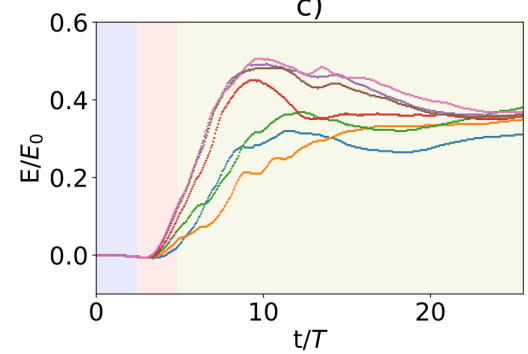

e)

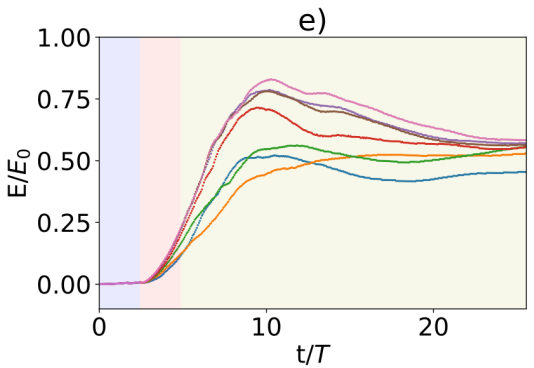

g)

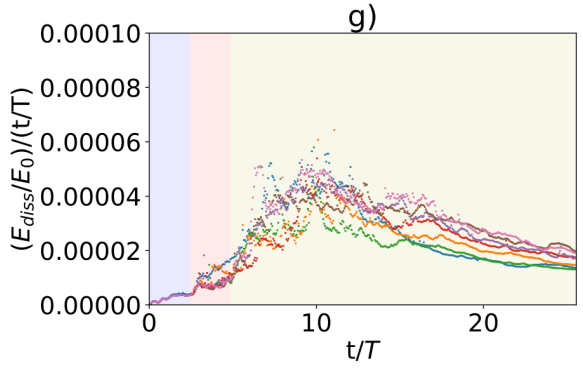

b)

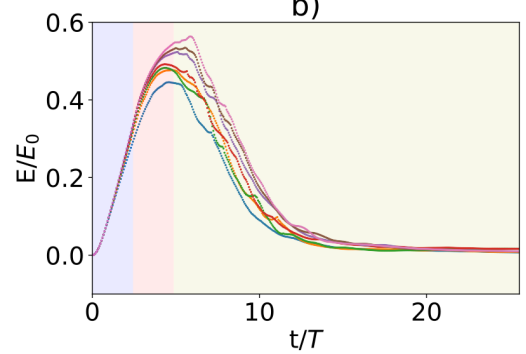

d)

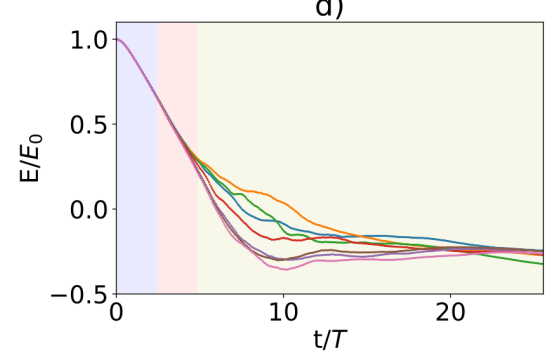

f)

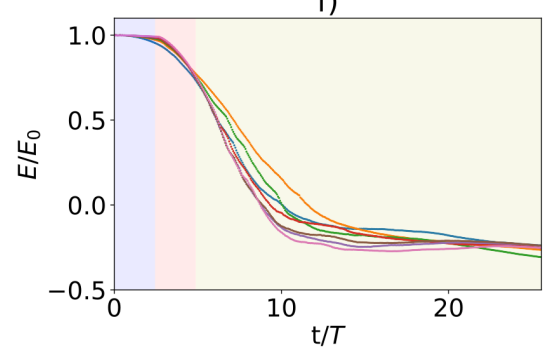

h)

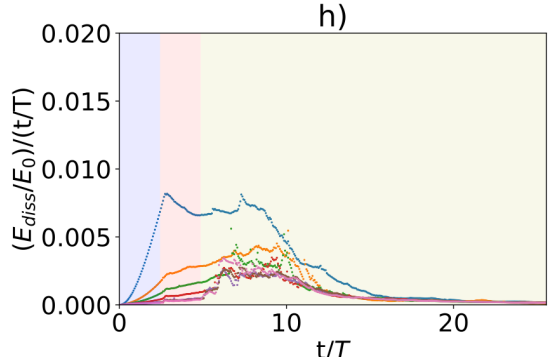

$\mathrm{b} / \mathrm{H}_{0}$

- 0.0

0.005

- 0.027

- 0.054

0.108

0.541

- 1.081

Figure 8. a) Kinetic energy evolution of water. b) Kinetic energy evolution of granular-fluid. c) Potential energy evolution of water. d) Potential energy evolution of granular-fluid. e) Total energy evolution of water. f) Total energy evolution of granular-fluid. g) Dissipation rate of water. h) Dissipation rate of granular-fluid. Results show a range of dimensionless slip lengths, for the initial condition where $\mu=0.1 \mathrm{~Pa} s$ and $H_{0}=18.5 \mathrm{~cm}$. 
gests that the wave is carrying sufficient energy to propagate significantly further, without considerable dissipation, if it were to continue in an infinite domain. Generally, the total energy of the water is greatest for lower friction conditions, with a few exceptions where an increase in total energy is observed for values of $b / H_{0}$ at $t / T \approx 20$. We hypothesize this to be a result of granular-fluid propagation: in these cases, snapshots and energy evolution plots demonstrate that the granular-fluid remains attached (or reattaches) to the bottom boundary, thus imparting more energy to the water in the near-field than simulations where the granular-fluid lifts off the tank bottom. This suggests that energy plots for the separate components, when considering the entire domain, cannot tell us all the information about the far-field wave if the gravity current does not behave in a consistent manner between simulations. For this reason, far-field wave gauges are used to reflect the relationship between the slip length and the total energy of the generated wave in the far-field, without considering the energy at the interaction or granular-fluid propagation zone.

In summary, the boundary condition influences the energy dissipated in the granularfluid on the slope prior to interaction, and hence the energy available for wave generation. It also determines the detailed mechanism by which this available energy is transferred to the generated wave. As viscosity is decreased, or initial column height is increased, the boundary condition has a less significant impact on the energy dissipation within the granular-fluid prior to impact. The boundary condition does, however, continue to affect the interaction dynamics (see Figure S3). Therefore under these conditions, despite similar overall energy in the granular-fluid at impact, significant discrepancies still exist between energy evolutions associated with different boundary conditions (see Figure $\mathrm{S} 4)$.

Understanding the energy transfer mechanisms also helps to interpret qualitative observations from Section 3.2 and quantitative results from Section 3.3.2, which showed that free-slip conditions (associated with efficient energy transfer and little to no wave breaking) overestimate near-field laboratory wave amplitudes, compared with partialslip conditions.

\subsubsection{Far-field dimensionless wave amplitude}

Figures 9 a-d show the influence of the slip condition and the boundary velocity, on the far-field wave amplitude (considered at $x_{1}=6 \mathrm{~m}$ from the shoreline) and maximum total energy of the water. These figures are for the initial conditions $H_{0}=18.5$ $\mathrm{cm}$ and $\mu=0.1 \mathrm{Pas}$. The far-field wave amplitude and the maximum total energy of the water follow an almost identical pattern of dependence on slip length. Between $b / H_{0}$ $=0$ and $b / H_{0}=0.1$, there is a sharp increase in both the maximum total energy of the water and the far-field amplitude. At $b / H_{0}>0.2$, this increase becomes more gentle. The maximum total energy of the water occurs at $t / T \approx 10$, suggesting that most of the initial energy transfer from the granular-fluid to the wave occurs in this time. Figure $9 \mathrm{~b}$ and Figure 9d show how maximum total energy of the water and maximum amplitude, respectively, vary with the dimensionless slope boundary velocity. As the boundary velocity increases, it is only once $u_{x, \text { boundary }} / u_{x, \max }>0.50$ that we observe a significant increase in energy transferred to the wave and likewise, a significant increase in the resulting wave amplitude. This result is surprising, since between $u_{x, \text { boundary }} / u_{x, \text { max }}>$ $=0$ and $u_{x, \text { boundary }} / u_{x, \max }=0.50$, we expect the largest change in the shape of the boundary layer, and thus energy dissipation. We therefore attribute this behavior to the change in interaction dynamics; as basal slip is increased, energy is more efficiently transferred into the total energy of the wave.

These observations enable us to quantitatively determine the relationship between slip length and energy transfer. For the initial conditions presented, we can infer for a given slip length, what the resulting energy transfer or far-field amplitude would be. Upon changing the initial conditions, if the influence of the slope boundary condition on the granular-fluid velocity profile decreases (i.e. viscosity decreases or column height increases), 
a)

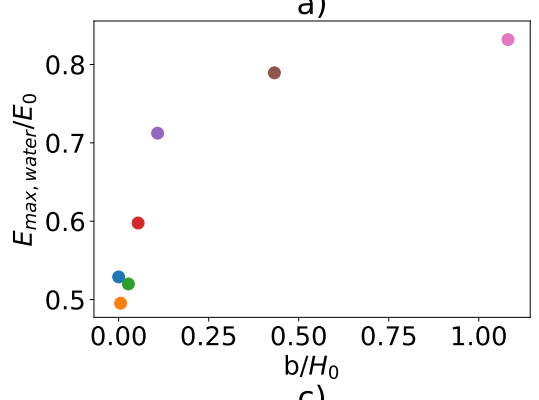

c)

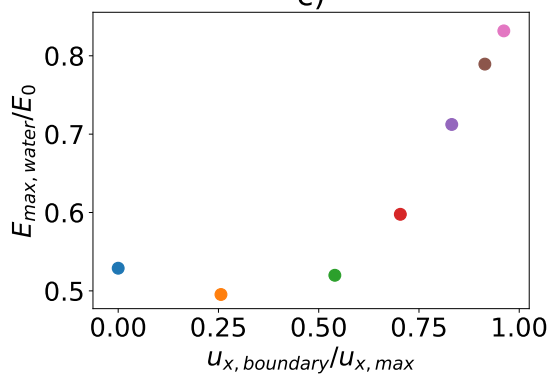

b)

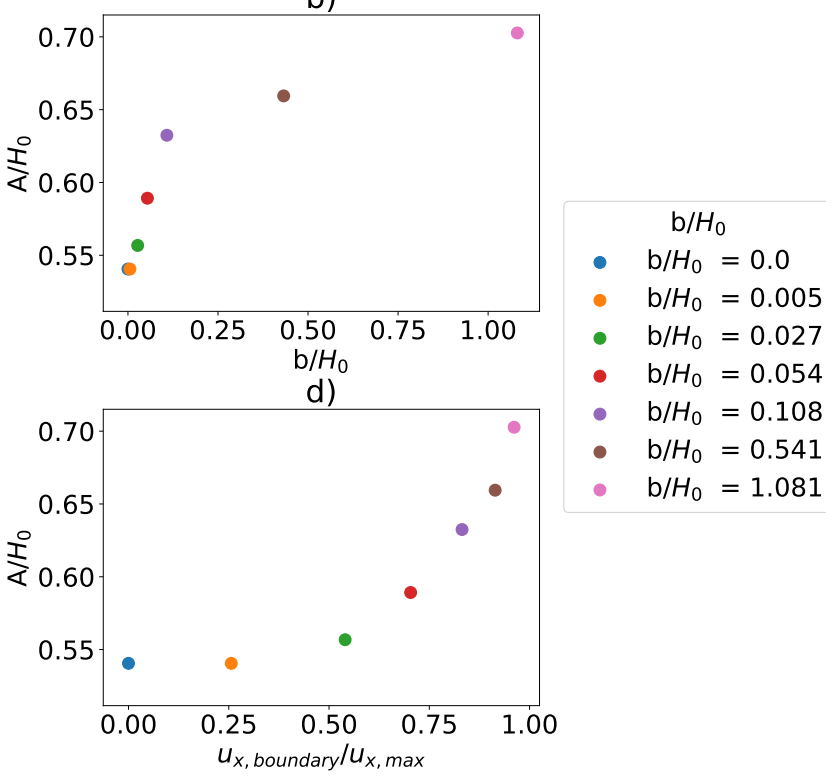

Figure 9. Effect of boundary condition on the far-field amplitude $\left(x_{1}=6 \mathrm{~m}\right.$ from the shoreline) and maximum total energy of the water. a) Total energy of water variation with slip condition. b) Dimensionless far-field ( $6 \mathrm{~m}$ from source) amplitude relative to the slip condition $b / H_{0}$. c) Total energy of water variation with dimensionless boundary velocity at impact $u_{\text {boundary }} / u_{\text {max }}$. d) Dimensionless far-field amplitude variation with $u_{\text {boundary }} / u_{\text {freestream }}$. These relationships are for $H_{0}=18.5 \mathrm{~cm}$ and $\mu=0.1 \mathrm{~Pa} \mathrm{~s}$.

the same relationships are generally observed, with less difference between maximum and minimum total energy or amplitude.

The overall energy dissipated in the interaction and wave generation process can be a result of viscous friction, air entrainment, mixing processes and the directionality of the granular-fluid front, which influences the amount of of overturning associated with the wave breaking. These observations highlight the importance of exploring the processes occurring at (and before) PDC-water interaction in more detail, in order to capture more accurate initial conditions when performing a numerical hazard assessment and exploring a wider range of possible scenarios. Accounting for processes such as mixing at the shoreline might also be a vital step in understanding the characteristics of associated tsunamis.

\section{Conclusions}

Numerical experiments on the entrance of fluidized granular flows into water have been carried out and compared against laboratory results, in order to explore how the slope boundary condition plays a role in determining the vertical $u_{x}$ velocity profile of the flow, the associated wave generation mechanism and the far-field wave characteristics.

It is shown that the boundary condition of the slope heavily determines to what extent the granular-fluid shears the water surface or propagates down-slope, which has important implications for the wave generation and breaking process. For low values of slip, the granular-fluid shears the water surface at impact, leading to considerable overturning and wave breaking at the interaction zone. For higher slip conditions, the granularfluid momentum is directed increasingly down-slope (beneath the water surface) and the 
overturning associated with the wave breaking decreases. It is concluded that high values of partial-slip lead to the closest qualitative representation of the experimental results.

We consider the relationship between the dimensionless product impulse parameter proposed by Bougouin et al. (2020) and the dimensionless wave amplitude $A / H_{i}$ (at $x=2.4 \mathrm{~m}$ from the interaction zone) and show that the numerical results display a good quantitative agreement with the laboratory experiments, helping to confirm our Newtonian fluid approximation. Partial-slip conditions appear to be a closer predictor of dimensionless wave amplitude than free-slip conditions, which is in agreement with the qualitative comparison.

Energy transfer is considered, in order to understand the far-field impact of the different slope boundary conditions and associated interaction dynamics in greater detail. The timing of the different phases of the simulation (e.g. granular-fluid propagation, impact, initial uplift, impact crater collapse and breaking) is inferred from our outputs. The importance of energy dissipation and transfer processes in influencing the wave characteristics is demonstrated, confirming why significant differences in near and far-field wave amplitudes are observed between slip conditions. We demonstrate a non-linear relationship between the dimensionless slip length (or basal slip velocity) and the maximum total energy of the water, as well as the far-field amplitude: increasing the boundary velocity at the time of impact makes no significant difference to the energy transfer in the resulting wave until $u_{x, \text { boundary }} / u_{x, \max }>0.5$. It is concluded that as basal slip is increased, energy is more efficiently transferred into the total energy of the wave.

When considering large scales, these observations may have significant implications for numerical simulations of PDC tsunami hazard. Firstly, these experiments confirm the assumption that a fluidized granular-flow can be modeled as a viscous Newtonian fluid, particularly in the context of wave generation. Using this assumption, our experiments demonstrate the importance of using an adequate boundary condition for the slope in order to capture the physics of wave generation and the associated far-field wave characteristics. Our results also highlight the sensitivity of the wave generation process to vertical variations in the horizontal velocity components within the granular-fluid, which is dependent on the relative importance of viscosity. This suggests that exploring the impact of vertical velocity stratification within highly-mobile PDCs may be an important next step when considering large-scale impacts of these flows with seawater. This would require the use of a multi-layer model. Furthermore, our results confirm that denserthan-water, fluidized granular-flows are capable of shearing the water surface and still generating waves of significant amplitude.

\section{Acknowledgments}

We would like to acknowledge the University of Auckland Doctoral Scholarship for its generous contribution of $\mathrm{PhD}$ fees and stipend of Lily Battershill. The project is also supported by the Marsden Fund Council from Government funding, managed by Royal Society Te Aprangi.

We thank Alexis Bougouin, Raphal Paris and Olivier Roche for the sharing of their experimental results, including the snapshots presented in Figure 2.

The authors also wish to acknowledge the use of New Zealand eScience Infrastructure (NeSI) high performance computing facilities, consulting support and/or training services as part of this research. New Zealand's national facilities are provided by NeSI and funded jointly by NeSI's collaborator institutions and through the Ministry of Business, Innovation and Employment's Research Infrastructure programme. https://www .nesi.org.nz.

Thanks also to the two anonymous reviewers whose insightful comments were invaluable in increasing the clarity and impact of this paper.

Basilisk is a freely available, multi-purpose tool to solve partial differential equations, developed by Popinet and collaborators (2013-2020). It has its own website, which provides general information including installation instructions and a tutorial: http:// 
www.basilisk.fr. The code contains solvers for Saint-Venant problems, the Navier-Stokes equations and more, see http://basilisk.fr/src/README. The Basilisk setup code used for the numerical simulations is freely available at https://doi.org/10.5281/zenodo .5601492 and the data used in the manuscript can be easily generated by running these scripts with the Basilisk software.

\section{References}

Andrews, B. J. (2019). Recognizing unsteadiness in the transport systems of dilute pyroclastic density currents. Bulletin of Volcanology, 81(2), 5.

Andrews, B. J., \& Manga, M. (2012). Experimental study of turbulence, sedimentation, and coignimbrite mass partitioning in dilute pyroclastic density currents. Journal of Volcanology and Geothermal Research, 225, 30-44.

Audusse, E. (2005). A multilayer Saint-Venant model: derivation and numerical validation. Discrete and Continuous Dynamical Systems - Series B, 5(2), 189214. doi: 10.3934/dcdsb.2005.5.189

Báez, W., de Silva, S., Chiodi, A., Bustos, E., Giordano, G., Arnosio, M., .. Groppelli, G. (2020). Pulsating flow dynamics of sustained, forced pyroclastic density currents: insights from a facies analysis of the Campo de la Piedra Pómez ignimbrite, southern Puna, Argentina. Bulletin of Volcanology, 82, $1-32$.

Baxter, P. J., Boyle, R., Cole, P., Neri, A., Spence, R., \& Zuccaro, G.

$(2005$, Apr 01). The impacts of pyroclastic surges on buildings at the eruption of the Soufrière Hills volcano, Montserrat. Bulletin of Volcanology, 67(4), 292313. Retrieved from https://doi.org/10.1007/s00445-004-0365-7 doi: 10.1007/s00445-004-0365-7

Bell, J. B., Colella, P., \& Glaz, H. M. (1989). A second-order projection method for the incompressible Navier-Stokes equations. Journal of Computational Physics, 85(2), 257283. doi: 10.1016/0021-9991(89)90151-4

Belousov, A., \& Belousova, B. V. (2000). Tsunamis generated by subaquatic volcanic explosions: unique data from 1996 eruption in Karymskoye Lake, Kamchatka, Russia. Pure and Applied Geophysics, 157(6-8), 11351143. doi: $10.1007 / \mathrm{s} 000240050021$

Belousov, A., Voight, B., \& Belousova, M. (2007). Directed blasts and blastgenerated pyroclastic density currents: a comparison of the Bezymianny 1956, Mount St Helens 1980, and Soufrière Hills, Montserrat 1997 eruptions and deposits. Bulletin of Volcanology, 69(7), 701-740.

Bonaccorso, A., Calvari, S., Garfì, G., Lodato, L., \& Patanè, D. (2003). Dynamics of the December 2002 flank failure and tsunami at Stromboli volcano inferred by volcanological and geophysical observations. Geophysical Research Letters, $30(18)$.

Bougouin, A., Paris, R., \& Roche, O. (2020). Impact of fluidized granular flows into water: implications for tsunamis generated by pyroclastic flows. Journal of Geophysical Research: Solid Earth, 125(5). doi: 10.1029/2019jb018954

Branney, M. J., \& Kokelaar, B. P. (2005). Pyroclastic density currents and the sedimentation of ignimbrites: Geological Society memoir. The Journal of Geology, 113(1), 115116. doi: 10.1086/427850

Breard, E., Dufek, J., Fullard, L., \& Carrara, A. (2020). The basal friction coefficient of granular flows with and without excess pore pressure: Implications for pyroclastic density currents, waterrich debris flows, and rock and submarine avalanches. Journal of Geophysical Research: Solid Earth, 125(12). doi: 10.1029/2020jb020203

Brodu, N., Delannay, R., Valance, A., \& Richard, P. (2015). New patterns in highspeed granular flows. Journal of Fluid Mechanics, 769, 218-228.

Carey, S., Sigurdsson, H., Mandeville, C., \& Bronto, S. (1996). Pyroclastic flows 
and surges over water: an example from the 1883 Krakatau eruption. Bulletin of Volcanology, 57(7), 493. doi: 10.1007/s004450050108

Cas, R. A., \& Wright, J. V. (1991). Subaqueous pyroclastic flows and ignimbrites: an assessment. Bulletin of Volcanology. doi: 10.1007/BF00280227

Center, N. G. D. $\quad$ (2006, Jul). Ngdc/wds global historical tsunami database. U.S. Department of Commerce. Retrieved from https://www.ngdc.noaa.gov/ hazard/tsu_db.shtml

Deike, L., Popinet, S., \& Melville, W. (2015). C Capillary effects on wave breaking. Journal of Fluid Mechanics, 769, 541569. doi: 10.1017/jfm.2015.103

Delannay, R., Valance, A., Mangeney, A., Roche, O., \& Richard, P. Granular and particle-laden flows: from laboratory experiments to field observations. Journal of Physics D: Applied Physics, 50(5), $053001 . \quad$ doi: 10.1088/1361-6463/50/5/053001

Dellino, P., Dioguardi, F., Doronzo, D. M., \& Mele, D. (2019). The rate of sedimentation from turbulent suspension: An experimental model with application to pyroclastic density currents and discussion on the grain-size dependence of flow runout. Sedimentology, 66(1), 129-145.

Dellino, P., Dioguardi, F., Doronzo, D. M., \& Mele, D. (2020). A discriminatory diagram of massive versus stratified deposits based on the sedimentation and bedload transportation rates. experimental investigation and application to pyroclastic density currents. Sedimentology, 67(4), 2013-2039.

Dellino, P., Zimanowski, B., Büttner, R., La Volpe, L., Mele, D., \& Sulpizio, R. (2007). Large-scale experiments on the mechanics of pyroclastic flows: Design, engineering, and first results. Journal of Geophysical Research: Solid Earth. doi: $10.1029 / 2006 J B 004313$

Dufek, J. (2016). The fluid mechanics of pyroclastic density currents. Annual Review of Fluid Mechanics. doi: 10.1146/annurev-fluid-122414-034252

Dufek, J., Ongaro, T. E., \& Roche, O. (2015). Pyroclastic density currents. The Encyclopedia of Volcanoes, 617629. doi: 10.1016/b978-0-12-385938-9.00035-3

Edmonds, M., \& Herd, R. A. (2005). Inland-directed base surge generated by the explosive interaction of pyroclastic flows and seawater at Soufriere Hills volcano, Montserrat. Geology, 33(4), 245-248.

Egorov, Y. (2007). Tsunami wave generation by the eruption of underwater volcano. Natural Hazards and Earth System Science. doi: 10.5194/nhess-7-65-2007

Esposti Ongaro, T., de'Michieli Vitturi, M., Cerminara, M., Fornaciai, A., Nannipieri, L., Favalli, M., ... others (2021). Modeling Tsunamis Generated by Submarine Landslides at Stromboli Volcano (Aeolian Islands, Italy): A Numerical Benchmark Study. Frontiers in Earth Science, 9, 274.

Fauria, K. E., Manga, M., \& Chamberlain, M. (2016). Effect of particle entrainment on the runout of pyroclastic density currents. Journal of Geophysical Research: Solid Earth, 121 (9), 6445-6461.

Fisher, R. V. (1979). Models for pyroclastic surges and pyroclastic flows. Journal of Volcanology and Geothermal Research, 6(3-4), $305318 . \quad$ doi: 10.1016/0377-0273(79)90008-8

Fornaciai, A., Favalli, M., \& Nannipieri, L. (2019). Numerical simulation of the tsunamis generated by the Sciara del Fuoco landslides (Stromboli Island, Italy). Scientific reports, $9(1), 1-12$.

Freundt, A. (2003). Entrance of hot pyroclastic flows into the sea: experimental observations. Bulletin of Volcanology, 65 (2), 144164. doi: 10.1007/s00445-002 $-0250-1$

Fries, A., Roche, O., \& Carazzo, G. (2021). Granular mixture deflation and generation of pore fluid pressure at the impact zone of a pyroclastic fountain: Experimental insights. Journal of Volcanology and Geothermal Research, 414, 107226.

Fritz, H. M., Hager, W. H., \& Minor, H.-E. (2003). ～Landslide generated impulse 
waves: hydrodynamic impact craters. Experiments in Fluids, 35(6), 520532. doi: 10.1007/s00348-003-0660-7

Fritz, H. M., Hager, W. H., \& Minor, H.-E. (2004). Near field characteristics of landslide generated impulse waves. Journal of Waterway, Port, Coastal, and Ocean Engineering, 130(6), 287302. doi: 10.1061/(asce)0733-950x(2004)130:6(287)

Giordano, G., \& De Astis, G. (2021). The summer 2019 basaltic Vulcanian eruptions (paroxysms) of Stromboli. Bulletin of Volcanology, 83(1), 1-27.

Giudicepietro, F., López, C., Macedonio, G., Alparone, S., Bianco, F., Calvari, S., ... others (2020). Geophysical precursors of the July-August 2019 paroxysmal eruptive phase and their implications for Stromboli volcano (Italy) monitoring. Scientific reports, $10(1), 1-16$.

Heller, V. (2009). Landslide generated impulse waves experimental results. Coastal Engineering 2008. doi: 10.1142/9789814277426_0109

Joubert, N., Gardin, P., Zaleski, S., \& Popinet, S. (2020). Modelling of mass transfer in a steelmaking ladle. In $C f d$ 2020.

Jutzeler, M., Manga, M., White, J., Talling, P., Proussevitch, A., Watt, S., ... Ishizuka, O. (2017). Submarine deposits from pumiceous pyroclastic density currents traveling over water: An outstanding example from offshore Montserrat (IODP 340). GSA Bulletin, 129(3-4), 392-414.

Legros, F., \& Druitt, T. (2000). On the emplacement of ignimbrite in shallowmarine environments. Journal of Volcanology and Geothermal Research, 95(14), 922. doi: 10.1016/s0377-0273(99)00116-x

Lube, G., Breard, E., Esposti-Ongaro, T., Dufek, J., \& Brand, B. (2020). Multiphase flow behaviour and hazard prediction of pyroclastic density currents. Nature Reviews Earth and Environment, 1(7), 348365. doi: 10.1038/s43017-020-0064-8

Lube, G., Breard, E., Jones, J., Fullard, L., Dufek, J., Cronin, S. J., \& Wang, T. (2019). Generation of air lubrication within pyroclastic density currents. Nature Geoscience, 12(5), 381386. doi: 10.1038/s41561-019-0338-2

Lube, G., Breard, E. C. P., Cronin, S. J., \& Jones, J. (2015). Synthesizing largescale pyroclastic flows: Experimental design, scaling, and first results from pele. Journal of Geophysical Research: Solid Earth, 120(3), 14871502. Ioi: $10.1002 / 2014 \mathrm{jb011666}$

Maeno, F., \& Imamura, F. (2011). Tsunami generation by a rapid entrance of pyroclastic flow into the sea during the 1883 Krakatau eruption, Indonesia. Journal of Geophysical Research: Solid Earth, 116(9), 1-24. doi: 10.1029/2011JB008253

Mattioli, G. S., Voight, B., Linde, A. T., Sacks, I. S., Watts, P., Widwijayanti, C., ... Williams, D. (2007). Unique and remarkable dilatometer measurements of pyroclastic flow-generated tsunamis. Geology. doi: 10.1130/G22931A.1

MiDi, G. (2004). On dense granular flows. The European Physical Journal E, 14 , 341-365.

Mohammed, F., \& Fritz, H. M. (2012). Physical modeling of tsunamis generated by three-dimensional deformable granular landslides. Journal of Geophysical Research: Oceans, 117(C11). doi: 10.1029/2011jc007850

Narcisse, Z., Dunkley, P., Edmonds, M., Herd, R., Talipova, T., Andrey, K., \& Nikolkina, I. $\quad(2004,01)$. Tsunami generated by the volcano eruption on July 12-13, 2003 at Montserrat, Lesser Antilles. Science of Tsunami Hazards, 22.

Nishimura, Y., Nakagawa, M., Kuduon, J., \& Wukawa, J. (2000). Timing and Scale of Tsunamis Caused by the 1994 Rabaul Eruption, East New Britain, Papua New Guinea. Tsunamis Advances in Natural and Technological Hazards Research, 4356. doi: 10.1007/1-4020-3331-1_3

Nomikou, P., Druitt, T., Hübscher, C., Mather, T., Paulatto, M., Kalnins, L., .. . others (2016). Post-eruptive flooding of Santorini caldera and implications for tsunami generation. Nature communications, 7(1), 1-10. 
Paris, R. (2015). Source mechanisms of volcanic tsunamis. Philosophical Transactions of the Royal Society A: Mathematical, Physical and Engineering Sciences, 373(2053), 20140380.

Popinet, S. (2003). Gerris: A tree-based adaptive solver for the incompressible Euler equations in complex geometries. Journal of Computational Physics, 190(2), 572-600. doi: 10.1016/S0021-9991(03)00298-5

Popinet, S. (2009). An accurate adaptive solver for surface-tension-driven interfacial flows. Journal of Computational Physics, 228(16), 58385866. doi: 10.1016/j.jcp .2009 .04 .042

Popinet, S. (2015). A quadtree-adaptive multigrid solver for the Serre-Green-Naghdi equations. Journal of Computational Physics. doi: 10.1016/j.jcp.2015.09.009

Popinet, S. (2020). A vertically-lagrangian, non-hydrostatic, multilayer model for multiscale free-surface flows. Journal of Computational Physics, 418, 109609. doi: $10.1016 /$ j.jcp.2020.109609

Popinet, S., \& collaborators. (2013-2020). Basilisk. http://basilisk.fr.

Robbe-Saule, M., Morize, C., Henaff, R., Bertho, Y., Sauret, A., \& Gondret, P. (2020). Experimental investigation of tsunami waves generated by granular collapse into water. Journal of Fluid Mechanics, 907. doi: 10.1017/jfm.2020.807

Roche, O., Gilbertson, M., Phillips, J., \& Sparks, R. (2002). Experiments on deaerating granular flows and implications for pyroclastic flow mobility. Geophysical research letters, 29(16), 40-1.

Roche, O., Montserrat, S., Niño, Y., \& Tamburrino, A. (2008). Experimental observations of water-like behavior of initially fluidized, dam break granular flows and their relevance for the propagation of ash-rich pyroclastic flows. Journal of Geophysical Research: Solid Earth, 113(B12).

Roche, O., Montserrat, S., Niño, Y., \& Tamburrino, A. (2010). Pore fluid pressure and internal kinematics of gravitational laboratory air-particle flows: Insights into the emplacement dynamics of pyroclastic flows. Journal of Geophysical Research: Solid Earth, 115(B9).

Rowley, P. J., Roche, O., Druitt, T. H., \& Cas, R. (2014). Experimental study of dense pyroclastic density currents using sustained, gas-fluidized granular flows. Bulletin of Volcanology. doi: 10.1007/s00445-014-0855-1

Scardovelli, R., \& Zaleski, S. (1999). Direct numerical simulation of free-surface and interfacial flow. Annual Review of Fluid Mechanics, 31(1), 567603. doi: 10 .1146/annurev.fluid.31.1.567

Schlichting, H., \& Gersten, K. (2016). Boundary-layer theory. Springer.

Smith, G., Rowley, P., Williams, R., Giordano, G., Trolese, M., Silleni, A., .. . Capon, S. (2020). A bedform phase diagram for dense granular currents. Nature communications, $11(1), 1-11$.

Smith, G. M., Williams, R., Rowley, P. J., \& Parsons, D. R. (2018). Investigation of variable aeration of monodisperse mixtures: implications for pyroclastic density currents. Bulletin of Volcanology, 80(8), 1-12.

Sparks, R., Wilson, L., \& Hulme, G. (1978). Theoretical modeling of the generation, movement, and emplacement of pyroclastic flows by column collapse. Journal of Geophysical Research: Solid Earth, 83(B4), 1727-1739.

Sulpizio, R., Dellino, P., Doronzo, D., \& Sarocchi, D. $\quad$ (2014). Pyroclastic density currents: state of the art and perspectives. Journal of Volcanology and Geothermal Research, 283, 36-65.

Thoraval, M.-J., Takehara, K., Etoh, T. G., Popinet, S., Ray, P., Josserand, C., ... Thoroddsen, S. T. (2012). Von Krmn Vortex Street within an Impacting Drop. Physical Review Letters, 108(26). doi: 10.1103/physrevlett.108.264506

Ui, T., Matsuwo, N., Sumita, M., \& Fujinawa, A. (1999). Generation of block and ash flows during the 1990-1995 eruption of Unzen Volcano, Japan. Journal of Volcanology and Geothermal Research, 89(1-4), 123-137.

Valentine, G. A. (2020). Initiation of dilute and concentrated pyroclastic currents 
from collapsing mixtures and origin of their proximal deposits. Bulletin of Volcanology, 82(2), 1-24.

Watts, P. (2003). Theoretical analysis of tsunami generation by pyroclastic flows. Journal of Geophysical Research. doi: 10.1029/2002JB002265

Waythomas, C. F., \& Watts, P. (2003). Numerical simulation of tsunami generation by pryoclastic flow at Aniakchak Volcano, Alaska. Geophysical Research Letters. doi: 10.1029/2003GL017220

Wilson, C. (1980). The role of fluidization in the emplacement of pyroclastic claws: An experimental approach. Journal of Volcanology and Geothermal Research, $8(2-4), 231-249$.

Zweifel, A., Hager, W. H., \& Minor, H.-E. (2006). Plane impulse waves in reservoirs. Journal of Waterway, Port, Coastal, and Ocean Engineering, 132(5), 358368. doi: $10.1061 /($ asce $) 0733-950 x(2006) 132: 5(358)$ 